\title{
III. Vor der Aufnahme von Schilumim- verhandlungen: Die alliierte und deutsche Perspektive
}

Die Alliierten verfolgten die wirtschaftliche Entwicklung in Deutschland mit größter Aufmerksamkeit. Doch während sie große Summen in die westdeutsche Wirtschaft pumpten, drohte der Abfluß von Geldern durch die jüdischen Restitutionsforderungen. Von den deutschen Behörden behindert und in langwierigen Gerichtsverfahren festgefahren, hing der Restitutionsprozeß weitgehend vom Rückhalt der Alliierten ab. Um diesen Prozeß zu beschleunigen, machten die Spezialisten der Jewish Restitution Successor Organization (JRSO) den Vorschlag, die Forderungen zu "globalisieren“. Demnach sollte die JRSO mit den Länderregierungen über die Zahlung einer globalen Summe für sämtliche unerledigten Restitutionsfälle verhandeln, deren Weiterbearbeitung dann im einzelnen den Ländern überlassen geblieben wäre.

Vier führende jüdische Persönlichkeiten, darunter Nahum Goldmann und JRSO-Direktor Benjamin B. Ferencz, unterbreiteten die Globalisierungsidee am 10. April 1950 dem amerikanischen Hochkommissar für Deutschland, John McCloy. ${ }^{1}$ McCloy nahm den Vorschlag mit Begeisterung auf und versprach, darüber mit den Ministerpräsidenten der Länder zu verhandeln. Er regte zudem an, die Globalisierung auf den Bereich der Entschädigungszahlungen auszuweiten, eine Idee, die von vornherein zum Scheitern verurteilt war, da dies die außerhalb Israels nicht zu bewerkstelligende Gründung einer Entschädigungsantragsstelle erfordert hätte. Es wäre äußerst schwierig gewesen, Antragssteller in mehreren Staaten in einer privaten Organisation zusammenzufassen und die globale Entschädigung dann unter ihnen aufzuteilen.

Die individuelle Entschädigung galt als weitere Belastung der deutschen Wirtschaft. In der amerikanischen Besatzungszone durch das später in ähnlicher Form auch in der britischen und französischen Zone übernommene Gesetz Nr. 59 geregelt, galt die individuelle Entschädigung zudem als unbefriedigend und diskriminierend. Die Länder forderten vom Bund die Übernahme der individuellen Entschädigung, doch ohne Erfolg. Eine Lösung des Problems war nicht in Sicht. Auch die Frage der „Dritten Masse“ blieb ungelöst.

Ein weiterer Problempunkt war der mit beinahe unüberwindlichen Schwierigkeiten verbundene Geldtransfer. Guthaben auf Sperrmarkkonten waren für im Ausland wohnhafte Anspruchsteller kaum verfügbar. Obwohl die Behörden gewisse Nachsicht walten ließen und DPs sowie aus anderen Ländern eingereisten jüdischen Antragsstellern die Wiedererlangung ihres Eigentums und damit die Ausfuhr gewisser Sperrmarkbeträge in Form von persönlichen Effekten ermög-

1 Memorandum und Sitzungsprotokoll über die Unterredung mit dem Hohen Kommissar vom 10. 4. 1950, ISA, 2387/22. 
lichten, blieb der größte Teil dieser Gelder auf deutschen Bankkonten blockiert. Das American Joint Distribution Committee (AJDC) und die Jewish Agency for Palestine (JAFP) durften gewisse Beträge für die Rehabilitation besonders bedürftiger Holocaust-Überlebender (Kranke, psychisch Geschädigte und schwere Sozialfälle) in Deutschland aufwenden. Doch die JAFP brauchte das Geld vor allem in Israel. Eine gewisse Umgehung der Geldausfuhrbeschränkungen stellte die Sondererlaubnis für Emigranten dar, Eigentum mitzunehmen, bei dem es sich in Wirklichkeit um JAFP-Güter handelte. Dies betraf vor allem vorfabrizierte Häuser, die als Emigranteneigentum deklariert, exportiert und dann im von Wohnungsnot geplagten jungen Staat Israel von der JAFP den jeweiligen Bedürfnissen entsprechend im Land verteilt wurden. ${ }^{2}$ Die Ausfuhr anderer, von den JAFP-Absorptionsagenturen dringend benötigter Güter ließen die alliierten Gesetze nicht zu. Die Briten errichteten zusätzliche Barrieren: Während sie vor dem israelischen Unabhängigkeitskrieg versuchten, den Geldstrom in Richtung jüdische Gemeinschaft in Palästina zu stoppen, zeigte sich ihre feindliche Haltung gegen Israel danach vor allem in den Bereichen Emigration und Geldtransfer.

\section{Der Weg zum Verhandlungstisch}

Der leitende JAFP-Delegierte Maurice M. Boukstein warnte die Israelis im Frühjahr 1950 in einem Schreiben vor der ungünstigen amerikanischen Einstellung zum jüdischen Restitutionsprogramm:

„In Deutschland arbeitet nicht nur die Zeit gegen uns, wie jedermann nun klar sein dürfte, sondern auch eine gewisse Leitlinie amerikanischer Politik, was so manchem unserer Freunde in Israel nicht bewußt ist. Denn während der amerikanische Steuerzahler aufgefordert wird, Deutschland mit Milliardenbeträgen zu überschütten, bemühen wir uns um das Gegenteil: Wir versuchen, Vermögen aus Deutschland abzuziehen [...] Das Restitutionsprogramm war deshalb bei den amerikanischen Behörden nie besonders beliebt. ${ }^{\text {"3 }}$

Selbst McCloy war nicht in der Lage, die für Israel ungünstigen Vorgaben der amerikanischen Außenpolitik zu ändern. Anläßlich eines Treffens mit Goldmann ließ er es bei einem Aide-mémoire bewenden: Wenn Israel sich nicht mit dem Rinnsal von Retributionsgeldern abfinden wolle, die weit hinter den Erwartungen zurückbleiben würden, dränge sich die Schlußfolgerung, daß direkte Gespräche mit Deutschland unerläßlich seien, immer mehr auf. ${ }^{4}$ Doch solange Deutschland unter alliierter Kontrolle stand, lag die höchste Entscheidungsgewalt bei den Regierungen der drei Großmächte.

Den jüdischen Organisationen stellten sich gleichzeitig mehrere Aufgaben: Sie mußten die Großmächte von der Notwendigkeit überzeugen, die Umsetzung der

2 Landauer an Greenbaum vom 8. 9. 1949, CZA, S35/148; Protokoll Nr. 38/5709 über die Sitzung der israelischen Regierung betr. Genehmigung für den Import von vorgefertigten Häusern aus Deutschland für Gelder der JRSO am 20. 9. 1949, ISÁ.

3 Boukstein, New York, an Lipschitz, Jerusalem, vom 24. 3. 1950, CZA, Z6/387.

4 Memorandum und Sitzungsprotokoll der Unterredungen des HICOG am 10.4. 1950, ISA; Landauer an Gerling vom 12.4. 1950, CZA, S 35/70. 
Retribution zu erleichtern. Den Deutschen war darüber hinaus die Unerläßlichkeit der Sühne für begangene Verbrechen vor Augen zu führen, und nicht zuletzt mußte die jüdische Öffentlichkeit davon überzeugt werden, daß der Staat Israel und die NS-Opfer dringend auf Mittel angewiesen waren und es deshalb keine Alternative zum direkten Dialog zwischen Juden und Deutschen gab. Offensichtlich waren dazu Anstrengungen sowohl in Israel als auch außerhalb Israels erforderlich. Dagegen bestanden auch keine grundsätzlichen alliierten Einwände. Folgende Notiz von L.B. Richards vom britischen Foreign Office mag dies veranschaulichen: „Adenauer hat den Opfern der NS-Verfolgung die Rückerstattung ihres Eigentums und Entschädigung versprochen, und es ist ein wichtiger Bestandteil der alliierten Politik, für die Einhaltung dieser Versprechen zu sorgen. ${ }^{5}$ Sein amerikanischer Kollege Geoffrey W. Lewis vom Büro für deutsche Angelegenheiten im amerikanischen Außenministerium legte die Umrisse eines amerikanischen Programms für die Bundesrepublik Deutschland dar, dessen Punkt c) mit „Gerechte Behandlung von Hitlers Opfern ohne Kompromisse" überschrieben war. ${ }^{6}$ In Kenntnis der alliierten Tagespolitik gegenüber der Bundesrepublik versuchten jüdische Vertreter die Alliierten davon zu überzeugen, daß die jüdischen Ansprüche durchaus mit ihr vereinbar seien. Das wichtigste - wahrscheinlich auch einzige - Druckmittel war das Argument, daß es den Deutschen zum Vorteil gereiche, den jüdischen Forderungen entgegenzukommen.

Unter den herausragenden jüdischen Exponenten haben insbesondere Goldmann und Blaustein diesen Hinweis bei verschiedenen Gelegenheiten sehr gezielt verwendet, aber auch $\mathrm{McCloy}$ machte davon in der als besonders dramatisch geltenden öffentlichen Erklärung über die europäische Einheit vor dem Pilgrims Club of Great Britain Gebrauch. ${ }^{7}$ Davon zeugt auch Blankenhorns Notiz zu McCloys Rede vom 4. April 1950.8

Goldmanns Strategie war es hingegen, dieses häufig verwendete Argument dem wichtigsten Zweck, dem Kampf um den Erhalt von Reparationen vorzubehalten. Darunter verstand man nicht kleinere Zuschüsse zur Deckung laufender Defizite im israelischen Staatshaushalt, sondern ein umfangreiches Hilfspaket zur nachhaltigen Lösung akuter wirtschaftlicher und sozialer Probleme des jungen Staates. Auch der israelische Außenminister Moshe Sharett beabsichtigte, den Wunsch des Westens auszunützen, die Bundesrepublik Deutschland in die westliche Staatengemeinschaft einzugliedern. Er erwog, die jüdische Öffentlichkeit für die Forderung von Reparationen zu mobilisieren und dafür zu sorgen, daß die „Absicht der Amerikaner, Deutschland wieder in die Völkergemeinschaft aufzunehmen “, den Protest der Weltöffentlichkeit hervorrufen werde. ${ }^{9}$

5 Notiz von L.B. Richards vom 11. 3. 1950, PRO, FO 371/82606.

6 Programm "National Conference of the United States in World Affairs" vom 4. 4. 1950, YIVO, AJC, RG 347, GEN-12, Box 179.

7 SCHWARTZ, From Occupation, S. 216.

8 Notiz Blankenhorns zur Rede McCloys vom 4. 4. 1950, BArch, N 1351, Bd. 3, 4.

9 Abteilung Vereinigte Staaten, Israelisches Außenministerium, an die Israelische Botschaft in Washington, D.C. vom 26. 5. 1950, ISA, 2482/13. 
Die Bedingungen für solche Kampagnen waren aber alles andere als günstig. Der Kalte Krieg war bereits in vollem Gange und beide Blöcke versuchten, die öffentliche Meinung in Deutschland mit der Gewährung von Vorteilen und der Beseitigung von Härten für sich zu gewinnen. Aber auch die Furcht vor neuem deutschen Nationalismus und Aggression klang noch nach, und die Alliierten beabsichtigten, „Deutschland so schnell wie möglich in Westeuropa und in einem westeuropäischen Verteidigungsbündnis einzubinden, um die Sicherheit zu garantieren".10 Nach dem Ausbruch des Koreakrieges spitzte sich die Lage in Deutschland weiter zu. Man fürchtete einen bewaffneten Konflikt auf deutschem Boden bzw. eine sowjetische Invasion.

Das westliche Werben um Deutschland war intensiver und großzügiger als das sowjetische: Die westlichen Großmächte verpflichteten sich zu einer Revision des Besatzungsstatuts, wenn auch mit Vorbehalten. Aus einem Entwurf geht hervor, daß die Alliierten auf ihr Entscheidungsrecht in gewissen Bereichen nicht verzichten wollten. Ein solcher Bereich betraf die jüdischen Belange. Auf die Frage des JRSO-Direktors Benjamin B. Ferencz, was denn mit "Machtvorbehalt“ gemeint sei, erwiderte Hochkommissar McCloy, „vor Korea wäre ich in der Lage gewesen, Ihnen zu sagen, daß es sich dabei um die exklusive Behandlung des Restitutionsbereichs durch uns handelt, doch jetzt kann ich Ihnen nicht mehr sagen, was es bedeutet". ${ }^{\prime 1}$

Die Gefahr war offensichtlich. Deutschland erwartete eine Lockerung des Besatzungsstatuts und die Bereitschaft, alliierte Gesetze und Bestimmungen zu befolgen, schwand. Doch Ferencz berichtete, bei den Alliierten herrsche deshalb keine Panik. McCloy sei dagegen, sich die Gunst Deutschlands teuer zu erkaufen. Seiner Meinung nach würde die Bundesrepublik den Westen dem Osten ohnehin vorziehen. ${ }^{12}$ Dessenungeachtet spürten jüdische Antragsteller eine Verhärtung auf deutschen Ämtern. Ein interner Briefwechsel im israelischen Außenministerium weist darauf hin, daß den Alliierten diese Verhaltensänderung in Deutschland nicht entgangen war. ${ }^{13}$

Auf der Tagesordnung des Außenministertreffens der drei westlichen Großmächte vom 20. September 1950 in New York fehlten die jüdischen Forderungen nach Entschädigung und Retribution. Sie wurden dem von den Vereinigten Staaten, Großbritannien und Frankreich gegründeten Dreierausschuß zur Behandlung deutscher Fragen mit der Bezeichnung "Intergovernmental Study Group“ (IGS) übertragen. Die Israelis und die jüdischen Antragsteller waren an der Beibe-

10 Memorandum McCloys für den Präsidenten betr. Situation in Deutschland vom 10. 9. 1950, Truman Library, President's Secretary Files, Subject File, Foreign Affairs (Germany), PSF File, Box 178.

11 Livneh an den Rechtsberater und die Wirtschaftsabteilung vom 7.11. 1950, ISA, 531/4, vgl. das Schreiben von Katzenstein an Landauer vom 29. 11. 1950, ISA 2482/13, worin die Besorgnis über die kühle Haltung der Alliierten gegenüber den jüdischen Ansprüchen zum Ausdruck kommt.

12 Ferencz an Eli Rock vom 27. 9. 1950, AJDC, 4264.

13 Maurice Fischer an Eli Rock vom 27. 9. 1950; Memorandum betr. Besuch des Kanadischen Botschafters, Lt. General Maurice Pope, vom 14. 12. 1950, ISA, 2538/22; Der Rechtsberater an Robinson, Livneh und die Politische Abteilung vom 20. 10. 1950, ISA, 531/4. 
haltung der obersten alliierten Aufsicht über die finanzielle Rehabilitation der Juden, einschließlich der speziellen alliierten Gerichtsstellen („Boards of Review“) mit Entscheidungsgewalt in strittigen Restitutionsfällen, interessiert, nicht zuletzt weil deutsche Gerichte jüdischen Antragstellern eher negativ gesonnen waren.14 Die Zuständigkeit für Restitution und Entschädigung lag in jenem Zeitraum, der zweiten Hälfte des Jahres 1950, noch bei den Ländern, die dem Druck der den jüdischen Ansprüchen negativ gegenüberstehenden Öffentlichkeit besonders stark ausgesetzt waren. Doch auch von der mit zahlreichen Beamten, die schon unter den Nationalsozialisten gedient hatten, besetzten Verwaltung konnten die jüdischen Antragsteller nicht viel erwarten. Auch Adenauers Bemühungen zur einheitlichen gesetzlichen Regelung der Entschädigungsfrage auf Bundesebene kamen nur schleppend voran und konnten erst nach der Unterzeichnung des Luxemburger Abkommens zwischen der Bundesrepublik Deutschland und dem Staat Israel umgesetzt werden. Das verabschiedete Gesetz entlastete die Länder und übertrug die Entschädigungspflicht dem Bund.

Die alliierte Politik gegenüber der Bundesrepublik zu Beginn der fünfziger Jahre stiftete in Israel einige Verwirrung. Die vermeintliche Nachsicht gegenüber Kriegsverbrechern erregte großen Unmut, und die Nachricht über die Wiederbewaffnung Deutschlands rief Angstgefühle wach. Das Wiederaufleben des Rechtsextremismus wurde als böses Omen gewertet. ${ }^{15}$ Die Veränderungen im Besatzungsstatut schließlich veranlaßten den Staat Israel, energische Schritte zur Umsetzung der Reparationsansprüche zu ergreifen. Das revidierte Besatzungsstatut eröffnete gewisse Chancen, rief gleichzeitig aber auch pessimistische Zukunftserwartungen und damit Anreize zum sofortigen Handeln hervor. Die israelischen und jüdischen Aktivitäten im Bereich Reparationen indes waren ungeordnet und oft sogar konfus.

Den ersten Vorstoß unternahm der stellvertretende Leiter des Deutschlandreferats im Außenministerium, Avigdor Dagan, mit einem Positionspapier, das auf Ben Gurions ausdrücklichen Wunsch verfaßt wurde. ${ }^{16}$ Darauf folgte am 2. Juni 1950 der Bericht des Leiters der politischen Sektion der Forschungsabteilung im israelischen Außenministerium, Boris Gurel, über seine Deutschlandreise. Gurel bewertete die junge Bundesrepublik als Sicherheitsrisiko für Israel, Europa und den Nahen Osten und empfahl, Deutschland in der Sicherheitsplanung des Staates Israel dementsprechend zu berücksichtigen. Zudem skizzierte er die Möglichkeit,

${ }^{14}$ Robinson an Shabtai Rosenne, vom November 1950, ISA, 2482/13; Adler-Rudel an Kreutzberger vom 24. 11. 1950, CZA, A140/58.

15 Ausarbeitung „Militärpolitische Grundlagen“" vom 8.11. 1950, BArch, N 1351/5; Der Rechtsberater an das Konsulat in München vom 12. 10. 1950, ISA, 2543/1; Niederschrift über eine Unterredung zwischen dem Ausschuss der Direktoren von JAFP und McCloy vom 16.10. 1950, ISA, 533/2; Protokoll Nr. 311/10 der Regierungssitzung, $\$ 53$, vom 10. 10. 1950, ISA, 7263/9; Der deutsche Außenminister an die Botschaft in Tel Aviv vom 19. 10. 1950, USNA, NND 832865, RG 84, Box 2, Tel Aviv, 321.9 Germany; Der Außenminister an diverse diplomatische Mitarbeiter vom 13.10. 1950, USNA, NND 832865 , RG 84, Box 5; Memorandum on Conversation, 18 October 1950, USNA, NND 832865, RG 466, HICOG Bonn, Classified General Records, Box 37; Ford an den Außenminister vom 10. 10. 1950, USNA, NND 83865, RG 84, Box 2, Tel Aviv, 321.9 Germany.

16 Interview mit Avigdor Dagan vom 9. 10. 1986, BGA, Oral History Division. 
daß sich Deutschland zu einer dritten Kraft an der Spitze der europäischen Staaten $z$ wischen der UdSSR und den USA entwickeln könne. Im Wettstreit der zwei Supermächte werde sich die Bundesrepublik dem antisowjetischen Lager anschließen und dadurch in die Lage versetzt, seine - militärischen und anderen Ziele durchzusetzen. Auch sei Deutschland, so Gurel, nach wie vor an Expansion interessiert, und der Nahe Osten müsse als eine ideale Region für expansionistische deutsche Schritte betrachtet werden. Weiter heißt es in Gurels Bericht, Deutschland werde im Nahen Osten als israelfeindlicher Faktor auftreten und die Araber in ihrem aggressiven Unterfangen unterstützen. Dem Staat Israel falle demnach die Aufgabe zu, das jüdische Volk gegenüber dem wiederaufstrebenden Deutschland zu verteidigen. Gurel unterstrich, daß Israel das Recht habe, Entschädigung für die Verbrechen Hitlerdeutschlands zu fordern. Als eine der siegreichen militärischen Kräfte des Zweiten Weltkriegs habe Israel zudem Anspruch auf Reparationen im eigentlichen Sinne des Wortes. Die jüdische Brigade aus Palästina, die im Zweiten Weltkrieg auf britischer Seite mitgekämpft habe, berechtige Israel dazu. Israel solle deshalb eine Militärdelegation nach Deutschland entsenden, um die israelische Militärmacht dort während der Besatzung und bei den zukünftigen Friedensverhandlungen zu vertreten. Des weiteren schlug Gurel die Gründung eines interministerialen Instituts für deutsche Angelegenheiten vor, zu dessen Aufgaben die Behandlung der Entschädigungsfrage und die Koordination einer präventiven, energischen Politik gegenüber Deutschland gehören sollten. ${ }^{7}$ Gurels Bericht war ein undatiertes und unsigniertes Schriftstück, vermutlich $\mathrm{Da}$ gans Bericht mit der Überschrift "Kernfragen zu Deutschland“ beigelegt. Dieser enthält eine dringende Warnung an die israelische Regierung: Deutschland sei eine Gefahr und seine politische Entwicklung müsse aufmerksam verfolgt werden. Israel solle alles in seiner Macht stehende tun, um seine materiellen Ansprüche gegenüber diesem Land zu realisieren. Diese beiden Dokumente vermitteln ein Gefühl der Dringlichkeit: Die Zeit sei knapp und falls Israel daran interessiert sei, Geld zu erhalten, bevor Deutschland eine Großmacht werde, müsse rasch gehandelt werden.

Um diese Dokumente richtig zu verstehen, muß man sich das unter dem Eindruck der jüngsten Geschichte herrschende Klima der Angst in Israel und die obsessiven Versuche vergegenwärtigen, aus der jüngsten Geschichte Schlußfolgerungen zu ziehen. Die Verfasser verkörperten die typische israelische und jüdische Geisteshaltung jener Zeit, und das Geschriebene reflektierte durchaus authentische Gefühle. Die Befürchtung, die Gunst der Stunde könnte ungenutzt verstreichen, spornte zum Handeln an. Doch, wie bereits erwähnt, waren die von den jüdischen Organisationen außerhalb Israels schon längst als unerläßlich erkannten, dem israelischen und jüdischen Denken aber nach wie vor suspekten, direkten Gespräche mit Deutschland eine Hürde auf dem Weg zur Lösung der Reparations- und Entschädigungsfrage. Um diese zu überwinden, setzte das israelische Außenministerium zusammen mit jüdischen Kreisen außerhalb des jüdischen Staates eine Kampagne in Gang, um solche Verhandlungen in die Wege zu leiten.

17 Forschungsabteilung an den Generaldirektor vom 12. 6. 1950, ISA, 2413/2. 
Der unmittelbare Auslöser für den israelischen Entschluß aktiv zu werden, waren die Beschlüsse des alliierten Außenministertreffens in New York vom September 1950, das „eine Reihe von Einigungen über Deutschland erzielte, zwecks Hebung des internationalen Ansehens und der internationalen Verantwortung der Bundesrepublik Deutschland und ihrer Regierung ". ${ }^{18}$ Die alliierten Außenminister einigten sich auf eine Beendigung des Kriegszustandes mit Deutschland, die Aufnahme diplomatischer Beziehungen zur Bundesrepublik sowie ihre Eingliederung in bestimmte internationale Organisationen. Für jeden dieser Schritte warben die Alliierten um israelische Unterstützung. Die alliierten Vorstöße führten zu einer gründlichen Überprüfung der Deutschlandpolitik im israelischen Außenministerium und später auch im Kabinett. In ihrem ersten Vorstoß suchten die Alliierten die Unterstützung Israels für die Aufnahme der Bundesrepublik Deutschland in die Organisation der UN für Erziehung, Wissenschaft und Kultur (UNESCO), die Weltgesundheitsorganisation (WHO) und die Ernährungs- und Landwirtschaftsorganisation der Vereinten Nationen (FAO). In einem Bericht an seine Vorgesetzten schrieb der britische Geschäftsträger in Tel Aviv, Sir K. Helm: „Ich teilte Herrn Sharett mit, daß ich durchaus Verständnis habe für die besondere Haltung der Juden und Israels bezüglich Deutschland und daß ich sie in unserer Note - Nr. 150 - berücksichtigt habe ${ }^{\alpha}{ }^{19}$ Ein Aide-mémoire ähnlichen Inhalts ging der israelischen Regierung am 23. Oktober von amerikanischer Seite zu. ${ }^{20}$ Am nächsten Tag setzten die drei Westmächte die israelische Regierung sodann in getrennten Botschaften über ihre Absicht in Kenntnis, den Kriegszustand mit der Bundesrepublik Deutschland zu beenden, und ersuchten die israelische Seite, ihrerseits ein entsprechendes Gesetz zu verabschieden. Daraus folgerte der Generaldirektor des israelischen Außenministeriums, Walter Eytan, daß die Zeit reif sei für die Errichtung einer besonderen Abteilung für deutsche Angelegenheiten in seinem Amt. Ob es der israelischen Diplomatie gefiel oder nicht, die Deutschlandpolitik erforderten volle Aufmerksamkeit. Mit halbherzigen Schritten waren die gesteckten Ziele nicht zu erreichen.

Die alliierten Noten zwangen das israelische Außenministerium zu der bislang ernsthaftesten Auseinandersetzung über die deutschlandpolitischen Zielsetzungen. Der amerikanische Geschäftsträger in Tel Aviv, Richard Ford, berichtete, der israelische Regierungsvertreter habe die Note über die Beendigung des Kriegszustands (mit Deutschland) „äußerst kühl“ empfangen, und bemerkte, daß „dieses Land jeden Vorstoß im Hinblick auf einen Frieden mit Deutschland in absehbarer Zukunft, gleichgültig von welcher Seite, mit größtem Argwohn begegne“, und riet, künftig behutsamer vorzugehen. ${ }^{21}$

18 Der Außenminister an diverse diplomatische Mitarbeiter vom 13. 9. 1950, USNA, Diplomatic Division, 321.9, Germany, RG-84, Box 5.

19 Note Nr. 150 der britischen Botschaft in Tel Aviv vom 23. 10. 1950, PRO, FO 371/85130.

20 Aide-mémoire der amerikanischen Botschaft in Tel Aviv an die israelische Regierung vom 23. 10. 1950, USNA, 321.9, Germany, RG-84, Box 5.

21 Richard Ford an den Außenminister vom 27. 10. 1950, USNA, 321.9, Germany, RG-84, Box 2. 
Zwischen Oktober 1950 und Februar 1951 wurde der zukünftige Kurs gegenüber Deutschland im israelischen Kabinett mehrfach diskutiert. Zu einer Beendigung des Kriegszustandes mit Deutschland konnte sich die Regierung nicht entschließen. ${ }^{22}$ Im Zentrum der äußerst stürmischen, emotionsgeladenen Debatten über Deutschland standen vor allem die finanziellen Ansprüche Israels und deren Umsetzung. ${ }^{23}$

Wieder versuchte der israelische Gesandte in den Beneluxländern, Michael Amir, mit provozierenden Denkanstößen Bewegung in die Sache zu bringen. In einer scharfen internen Notiz bezichtigte er die israelische Politik der Doppelzüngigkeit, indem er darauf verwies, daß Österreich und Polen von Israel mit Nachsicht behandelt würden, obwohl deren Bürger nicht weniger antisemitisch eingestellt seien als die Deutschen. Er warf daher die Frage auf, warum Israel diese Staaten anerkenne und die Bundesrepublik nicht. Den israelischen Boykott Deutschlands bezeichnete er als „Donquichotterie“ und ging davon aus, daß Bonn am Gespräch mit Israel interessiert und zudem bereit sei, die Verbrechen der Nationalsozialisten zu verurteilen und die Juden zu entschädigen. Diesmal fielen Amirs Worte auf fruchtbaren Boden. ${ }^{24}$

Am 27. November 1950 richtete der Rechtsberater im israelischen Außenministerium, Shabtai Rosenne, ein umfassendes Memorandum an Außenminister Sharett und Generaldirektor Eytan, offenbar mit stillschweigender, wenn nicht aktiver Unterstützung weiterer hoher Beamter des Außenministeriums. Darin analysierte er die Rechtsgrundlage und Durchsetzungsmöglichkeit der israelischen Ansprüche und warnte vor zu raschen Erfolgen der westdeutschen Politik und davor, daß Änderungen im Besatzungsstatut zum Verlust des israelischen Konsulats in München führen könnten. Des weiteren betonte er den Standpunkt, daß zwischen Israel und der Bundesrepublik Deutschland kein Kriegszustand bestehe und Israel somit nicht beenden könne, was von vornherein gar nicht existiere. Dieser Umstand sei nicht öffentlich einzugestehen, dürfe aber nicht daran hindern, weitere Maßnahmen zur Aufnahme deutsch-israelischer Verbindungen zu treffen. Hierzu empfahl er die Errichtung einer israelischen Mission - selbst mit diplomatischem Status, falls unumgänglich - in Deutschland und die Aufnahme direkter Reparationsverhandlungen. ${ }^{25}$

Zehn Tage später richtete der Direktor der Westeuropaabteilung, Gershon Avner, ein Schreiben an Generaldirektor Eytan, in dem es unter anderem hieß: „Die Erkenntnis, zu der wir nun alle gelangt sind, nämlich daß ein gewisses $\mathrm{Ma} ß$ an direkten Beziehungen mit der deutschen Regierung für die Umsetzung unserer Ansprüche unumgänglich ist, muß der Regierung klar vor Augen geführt werden. Die Errichtung einer Mission in Deutschland ist nicht gleichbedeutend mit der offiziellen Anerkennung Westdeutschlands." 26

22 Ford an den Außenminister vom 3.11. 1950, USNA, 321.9, Germany, RG-84, Box 2; HAARETZ (Tel Aviv) vom 12.11. 1950.

23 Interview mit Walter Eytan 1987, BGA, Oral History Division.

24 Amir an die Abteilung für Westeuropa vom 13.11. 1950, ISA, 2417/4.

25 Rosenne an den Außenminister und den Generaldirektor vom 27. 11. 1950, ISA, 2445/6.

26 Gershon Avner an den Generaldirektor vom 12. 12. 1950, ISA, 2445/6. 
Nicht alle hochrangigen Beamten im Außenministerium teilten jedoch diese Meinung, nicht alle waren davon überzeugt, daß sich Israel und Deutschland nicht im Kriegszustand befanden, und nicht alle waren zu direkten Verhandlungen mit Bonn bereit. Rosenne bestand darauf, die von ihm formulierten Fragen dem Kabinett vorzulegen, war aber nicht besonders optimistisch hinsichtlich der zu erwartenden Entscheidung: „Ich fürchte, nur eine Minderheit wird mit Verstand entscheiden. Die Mehrheit wird sich wieder von Emotionen und dem Verlangen nach der Begleichung irrelevanter historischer Rechnungen leiten lassen. ${ }^{27}$ Stellvertretend für Außenminister Sharett präsentierte Generaldirektor Eytan dem Kabinett fünf Alternativen: 1. keine israelische Antwort auf die alliierten Noten; 2. Kenntnisnahme dieser Noten; 3. die Großmächte informieren, daß Israel nicht bereit sei, den Kriegszustand mit Deutschland zu beenden; 4. eine Kombination von Punkt 2 und 3; 5. zwei separate Noten, von denen die erste eine Kombination von Punkt 2 und 3 darstellte und die zweite den israelischen Anspruch auf Reparationen präsentierte. In einem Schreiben an den Kabinettssekretär bekräftigte Eytan die Unumgänglichkeit des direkten Dialogs mit Deutschland und schlug die Entsendung einer Ad-hoc-Mission zum Zweck von Reparationsverhandlungen vor. ${ }^{28}$

Das Kabinett erwies sich als beschlußunfähig, und der sonst als pragmatisch geltende Ben Gurion machte sogar den Vorschlag, rückwirkend auf den 14. Mai 1948, den Tag der Gründung des Staates Israel, Deutschland den Krieg zu erklären.29 Dieser Vorschlag sorgte für Aufruhr im Außenministerium und verschwand rasch von der Tagesordnung. Am 3. Januar 1951 fällte das Kabinett den Beschluß, die Entsendung einer Mission und direkte Gespräche mit Deutschland gemäß den Plänen des Außenministeriums abzulehnen. Fünf Minister stimmten für diese Pläne, fünf dagegen. Statt dessen erfolgte ein Appell an die Besatzungsmächte, die Rückerstattung jüdischen Eigentums und Entschädigung zu garantieren.

In einer internen Beratung beschloß das Außenministerium zwei Tage später, die drei alliierten Noten zu beantworten und eine weitere Note an alle vier Besatzungsmächte anzufügen. In der Antwort auf die alliierten Noten vom 24. Oktober 1950 stellte das israelische Außenministerium fest, daß "die besondere Lage, in der sich dieser Staat befindet, keine Maßnahmen gemäß der von den westlichen Großmächten vorgeschlagenen Leitlinien rechtfertigen". Israel behalte sich seine eigenen Positionen, Rechte und Ansprüche bezüglich Deutschland vor. ${ }^{30} \mathrm{Zu}$ direkten Gesprächen mit Deutschland konnten sich die israelischen Entscheidungsträger immer noch nicht durchringen. Sie setzten weiterhin darauf, daß eine dritte Partei sich zur Durchsetzung der israelischen Forderungen bereit finden würde.

27 Rosenne an Robinson vom 13.12. 1950, ISA, 344/15.

28 Der Generaldirektor des israelischen Finanzministeriums an den Kabinettssekretär vom 17. 12. 1950, ISA, 2417/1.

29 Der Generaldirektor des israelischen Finanzministeriums an den Justizminister vom 27. 12. 1950, BGA, Correspondence Fíle 1950; Das Büro des Rechtsberaters an den Generaldirektor vom 2. 1. 1951, ISA, 2413/2.

30 News Bulletin to the Diplomatic Missions Nr. 186 vom 9. 1. 1951. 
Im Rahmen der Debatte über die Zukunft des israelischen Konsulats in München machte der amerikanische Hochkommissar John McCloy unter anderem den Vorschlag, daß Israel und die Bundesrepublik Deutschland diplomatische Beziehungen aufnehmen sollten. Diese Anregung erfolgte, nachdem die Militärbehörden die diplomatischen Missionen in Westdeutschland dazu aufgefordert hatten, ihre diplomatischen Vertretungen in Bonn zu akkreditieren, um den diplomatischen Status der Bundesrepublik zu erhöhen, und die israelische Regierung daraufhin erwogen hatte, ihre unter der Bedingung der ausschließlichen Akkreditierung bei den Besatzungsbehörden eröffnete Konsularvertretung zu schließen. Um einen solchen Schritt zu verhindern, nahm McCloy Verhandlungen über den zukünftigen Status des Konsulats auf, wobei er unter anderem obigen Vorschlag unterbreitete. Die Aufnahme diplomatischer Beziehungen zwischen den beiden Staaten hätte der alliierten Ziesetzung einer Erhöhung des internationalen Status der Bundesrepublik Deutschland zweifellos gedient. Doch das State Department wagte es angesichts der israelischen Position nicht, einen solchen Vorschlag offiziell zu machen, und fand schließlich einen anderen Ausweg aus dem Dilemma: Das israelische Konsulat blieb beim obersten Militärkommando und später, bis zur Eröffnung der Kölner Mission, beim Hochkommissar persönlich akkreditiert. $^{31}$

In einem Bericht an den amerikanischen Außenminister über die politischen Positionen in Israel zur „Deutschlandfrage" wies der amerikanische Geschäftsträger in Tel Aviv, Richard Ford, auf die Haßgefühle und deren politische Ausschlachtung durch die linke Opposition hin. Diese versuche, die Regierung in ihrer zunehmenden, wenn auch noch inoffiziellen, prowestlichen Neigung dadurch bloßzustellen, daß sie ihr vorwerfe, die Identifizierung mit dem Westen komme indirekt einem Bündnis mit den verhaßten Deutschen gleich. ${ }^{32}$ Unter solchen Bedingungen, folgerte Ford, könne es sich keine israelische Regierung leisten, diplomatische Beziehungen mit Deutschland aufzunehmen. McCloys Vorschlag sei deshalb unklug gewesen. Die israelische Regierung sei ohnehin schon in einer schwierigen Lage und jeder zusätzliche Druck werde ihr graduelles Abrücken von der neutralen Haltung zugunsten einer erklärten prowestlichen Ausrichtung negativ beeinflussen, meinte Ford. ${ }^{33}$

Die Wiederbewaffnung Deutschlands aufgrund der Brüsseler NATO-Konferenz im Dezember 1950, auf der Vorschläge für einen westdeutschen Beitrag zur Verteidigung Europas zur Debatte standen, alarmierte die Israelis und bot sowohl der linken als auch der rechten Opposition in Israel Gelegenheit zur Attacke gegen die Regierung. Regierungschef David Ben Gurion, der eine Politik der vorsichtigen Annäherung an den Westen verfolgte, kam die hitzige parlamentarische Debatte zu diesem Thema am 10. Januar 1951 höchst ungelegen. Er konnte die Verurteilung der Bewaffnung der Bundesrepublik nicht verhindern, und die linke Opposition mußte ihrerseits eine Verurteilung der Bewaffnung der DDR hinneh-

31 JELINEK, Like an Oasis, S. 86-88.

32 Ford an den Außenminister vom 31. 12. 1950, USNA, Tel-Aviv 321.9. Germany, RG-84, Box 2.

33 Ebd. 
men. In einer an alle Parlamente der Welt gerichteten Resolution drückte die Knesset ihre Besorgnis über die Wiederaufnahme Deutschlands in die Völkerfamilie aus, verurteilte die Wiederbewaffnung Deutschlands und appellierte an die Großmächte, ihrer „ursprünglichen Politik“ treu zu bleiben. Am Vortag der Knessetdebatte berichtete der amerikanische Geschäftsträger in Tel Aviv erneut über den „praktisch in allen Bevölkerungsschichten tief verwurzelten virulenten Haß gegen Deutschland“. Zwei Tage danach informierte er seinen Chef darüber, daß die Regierung vor dem „lautstark vorgebrachten späten“ antideutschen Ressentiment kapituliert habe. Angesichts der von $\mathrm{McCloy}$ erwogenen Amnestie für die in Landsberg einsitzenden Kriegsverbrecher erreichte der öffentliche Unmut in Israel dann einen neuen Höhepunkt.

Aus Furcht vor Attacken auf die USA und den Westen verhinderte die Regierungskoalition weitere Debatten über die deutsche Wiederbewaffnung und die Amnestie von Kriegsverbrechern im Knessetplenum und im parlamentarischen Ausschuß für auswärtige Angelegenheiten und Verteidigung. Im Bericht der amerikanischen Botschaft hieß es unter anderem: „Angesichts des weitverbreiteten und für die öffentliche Meinung in Israel so charakteristischen Hasses gegen Deutschland und alle Deutschen war die Resolution gegen die Wiederbewaffnung Deutschlands vom 10. Januar 1951 unvermeidlich. ${ }^{\text {34 }}$ Der amerikanische Botschafter wurde ins israelische Außenministerium zitiert, und Außenminister Sharett überreichte ihm einen scharfen Protest gegen die Amnestie der Kriegsverbrecher. Mündlich bemerkte Sharett, die Regierung sei in der Lage, organisierten öffentlichen Protest zu stoppen, jedoch nicht spontane Gefühlsausbrüche. ${ }^{35} \mathrm{Die}$ israelische Regierung war nicht erfreut über die Reaktion der Öffentlichkeit zur Deutschlandpolitik des Westens, da sie die Opposition begünstigte. Die öffentliche Meinung hatte die Regierung wider besseres Wissen zum Handeln gezwungen. Doch dies war nur ein Vorgeschmack auf spätere Versuche, die Deutschlandpolitik zu beeinflussen. Gewisse Berufsdiplomaten und vermutlich auch Politiker ahnten die unvermeidlichen Folgen der sich abzeichnenden Umwälzungen in Europa für Israel und leiteten daraus, ganz im Gegensatz zur breiten israelischen und jüdischen Öffentlichkeit, schon früh eine Notwendigkeit direkter Gespräche mit Deutschland ab. Doch die Regierung mußte der allgemeinen Stimmung aus politischen Gründen Rechnung tragen. Dazu äußerte sich der amerikanische Botschafter in Israel wie folgt: „Die Regierung hat angesichts der herrschenden politischen Umstände keine Alternative zu den obengenannten Schritten [die politischen Aktivitäten gegen die Wiederbewaffnung Deutschlands]: Diese bezwecken klar die Besänftigung der Emotionen."36 Die heftige israelische Reaktion auf die Wiederbewaffnung Deutschlands wurde auch von jenen amerikanischen und britischen Stellen registriert, die sich der Notwendigkeit bewußt waren, die israe-

34 Edward W. Holmes an den Botschafter vom 9.3. 1951, USNA, RG-84, Tel Aviv, Israel Consular Post, 1950-52; vgl. Edward W. Holmes an den Außenminister vom 9. 3. 1951, USNA, Tel Aviv, 321.9, Germany, RG-84, Box 5.

35 Protokoll der Zusammenkunft vom 12. 3. 1951, ISA, 2418/6a.

36 Monett B. Davis an den Außenminister vom 12. 3. 1951, USNA, 321.9, Germany, RG-84, Box 5 . 
lische (und jüdische) Öffentlichkeit im Rahmen einer weitreichenden Strategie zur innen- und außenpolitischen Festigung der Bundesrepublik zufriedenzustellen.

Die andauernde, äußerst emotional geführte Debatte über die Deutschlandpolitik in jüdischen und israelischen Kreisen fand ihren Ausdruck auch im diplomatischen Bereich. Der israelische Vertreter bei den Vereinten Nationen, Gideon Rafael, warnte den israelischen Botschafter in Washington, Israel und die USA befänden sich auf Kollisionskurs. Um einen solchen Zusammenstoß zu verhindern und die echte Freundschaft zwischen den beiden Staaten zu erhalten, so Rafael, sollte Israel die jüdische Öffentlichkeit in den USA mobilisieren. Wenn es um die Amnestie und die Aussetzung der Todesstrafe für Kriegsverbrecher gehe, „dürfe es auch unter Freunden mal zu einer Diskussion kommen ". ${ }^{37}$ Der israelische Gesandte in Großbritannien, Michael R. Kidron, berichtete über die Deutschlanddebatte in Großbritannien und über die starken antideutschen Emotionen in diesem Land. ${ }^{38}$ Solche Schreiben und Berichte waren keine Ausnahme. Sie veranschaulichten die allgemeine Stimmungslage. Dessenungeachtet wünschten Diplomaten die Behandlung der Reparationsfrage angesichts der stattfindenden Veränderungen im politischen Umfeld zu beschleunigen, während die von einem engeren nationalen Gesichtskreis ausgehenden Politiker die internationalen Entwicklungen mit einer Mischung von Gleichgültigkeit und Gelassenheit verfolgten. Der Versuch der westlichen Alliierten, das Ansehen der Bundesrepublik Deutschland mit israelischer Unterstützung zu erhöhen, beruhte auf mangelnder Kenntnis des israelischen Standpunkts und zeugte von der Notwendigkeit, die westliche Öffentlichkeit darüber aufzuklären. Insofern erfüllten die Proteste in Israel ihren Zweck, obwohl sie nicht der offiziellen Linie entsprachen. Die Bemühungen der israelischen Regierung, direkte Gespräche mit Deutschland durch die Mobilisierung der Supermächte, die UNO, durch den Internationalen Gerichtshof in Den Haag oder mittels eines eigens zu gründenden internationalen Komitees zu umgehen, waren fruchtlos. Bei allem Verständnis der Alliierten für die israelische Haltung - sie widersprach der dem jüdischen Staat zugedachten Rolle. Dies geht aus den alliierten Noten deutlich hervor. Für Israel und die jüdische Welt gab es keine Alternative zum schmerzlichen Weg.

Auch die deutsche Seite bewegte sich, wie erwähnt, nur zögernd auf die jüdische Seite zu. Nachdem seine Initiative vom Herbst 1949 gescheitert war, schenkte Konrad Adenauer den jüdischen und israelischen Angelegenheiten im folgenden Jahr nur noch geringe Aufmerksamkeit. Doch ausgerechnet das Jahr 1950 war international besonders ereignisreich, und die internationale Bedeutung der Bundesrepublik erhielt durch den Koreakrieg starken Auftrieb. Demgegenüber spielte die jüdische Problematik auf weltpolitischer Ebene eine untergeordnete Rolle, was den Kanzler aber nicht von Versuchen abzuhalten schien, die internationale Position der Bundesrepublik mit jüdischer Hilfe weiter zu verbessern. So wandte er sich unter Umgehung von Hochkommissar McCloy mit einer Botschaft an die amerikanische Öffentlichkeit, in der er Abscheu über die NS-Greuel bekundete,

37 Rafael an Eban vom 19. 2. 1951, ISA, 2445/6.

38 Kidron an Sharett vom 22. 2. 1951, ISA, 2445/6. 
den Antisemitismus und den Nationalismus verurteilte und sich für eine vollständige Rückerstattung des jüdischen Eigentums aussprach. Dieser Vorstoß war kein Einzelfall. Adenauers Politik zeugt von weiteren Versuchen, amerikanische Juden für deutsche $Z$ wecke einzuspannen. Vom besonderen Einfluß der amerikanischjüdischen Gemeinschaft auf die amerikanische Politik überzeugt, erachtete er die Zufriedenstellung dieser Gemeinschaft und die Gewinnung ihres Vertrauens als Möglichkeit zur Förderung deutscher Interessen. ${ }^{39}$

Die finanziellen und ökonomischen Bedürfnisse der Bundesrepublik waren enorm. Neben der Versorgung der Bevölkerung mußte auch die Unterstützung der Bedürftigen (Flüchtlinge, Vertriebene, Evakuierte etc.), der wirtschaftliche Wiederaufbau, die Finanzierung der Besatzungsarmeen, die Wiederbewaffnung und die Begleichung der Vorkriegsschulden sichergestellt werden. Es verwundert also nicht, daß die Geltendmachung der jüdischen Ansprüche mit größten Schwierigkeiten verbunden war, die den gesamten Retributionsprozeß in Deutschland überschatteten. Sie sind deshalb bei der Interpretation der deutschen Politik stets mitzuberücksichtigen. Auf jüdischer bzw. israelischer Seite spornte die latente Angst vor einem wiederbewaffneten Deutschland, einem Bündnis zwischen Deutschland und der arabischen Welt, einer feindseligen Politik gegenüber dem Staat Israel und die zunehmende Bedeutung der Bundesrepublik Deutschland im europäischen Verteidigungssystem zum Handeln an. Der Zusammenhang zwischen dem Wiederaufstieg Deutschlands und der schwindenden Hoffnung auf eine Erfüllung der jüdischen Forderungen schien offensichtlich. Die jüdische Seite wähnte sich unter Zeitdruck. Man fürchtete, die sich bietende Gelegenheit zu verpassen. Doch materielle Interessen sind bekanntlich oft stärker als politische Sachzwänge: Die kommerziellen und wirtschaftlichen Bedürfnisse Israels und Deutschlands schienen für eine Vertiefung der gegenseitigen Beziehungen zu sprechen, wie sich auf anderer Ebene bereits zeigte.

\section{Kommerzielle und wirtschaftliche Bedürfnisse}

Die Ausfuhr deutscher Produkte nach Palästina wurde bereits 1946 wieder aufgenommen. Neueinwanderer aus Deutschland, vor allem DPs, und jüdische Einwanderer aus anderen Ländern, die sich zu Restitutions- bzw. Entschädigungszwecken kurzfristig in Deutschland aufgehalten hatten, führten deutsche Produkte als persönliche Effekten in Israel ein und verkauften sie anschließend auf dem örtlichen Markt. Israelische Firmen erzielten hohe Profite durch die Einfuhr und den Verkauf von als Einwanderereigentum deklarierten deutschen Erzeugnissen. Damit wurde nicht nur der Handelsboykott, sondern auch der israelische Zoll umgangen, jedenfalls solange bis die Besatzungsmächte und die israelischen Behörden Maßnahmen ergriffen, um den unter dem Deckmantel persönlicher Effekten von Einwanderern verborgenen deutschen Export nach Israel zu beschränken oder ganz zu unterbinden. Diesem Export haftete ein schaler Beigeschmack

39 William Donovan an Henry A. Byroad vom 30. 3. 1950, USNA, 762A.13/3-3050. 
an, da er teilweise mit Schwarzgeld finanziert wurde. Gelegentlich wurde auch geschmuggelt. Dabei wurden Gelder aus Entschädigungs- und Restitutionsgeldern zum Einkauf deutscher Güter verwendet. Obwohl solche Gelder auf Sperrmarkkonten überwiesen wurden und nur ein Bruchteil davon direkt verfügbar war, konnten Sperrmarkbeträge mit einem gewissem Verlust in Hartwährung oder DM umgetauscht und direkt verwendet werden, etwa zum Einkauf billiger Ware in Deutschland, die dann mit ansehnlichem Profit ins güterhungrige Israel geschmuggelt wurde. Trotz Boykotts gab es auch zahlreiche amtliche Einfuhrbewilligungen für deutsche Güter.

AJDC, JAFP und ähnliche Organisationen deckten ihren Güterbedarf teils mit Importen aus Deutschland, der vor allem mit JRSO-Geldern finanziert wurde, und auch israelische Regierungsämter schreckten keineswegs davor zurück, aus Preis- und/oder Qualitätsgründen deutsche Produkte anderen Importprodukten vorzuziehen. Man kann davon ausgehen, daß die israelische Armee zu den Importeuren aus Deutschland zählte. Zur amtlichen, von der JRSO finanzierten Einfuhr deutscher Erzeugnisse zählten etwa die bereits erwähnten vorfabrizierten Häuser. Bis Oktober 1953 importierte allein die JAFP aus Deutschland vorfabrizierte Häuser im Wert von DM 13165000 , landwirtschaftliches Gerät und Dünger für DM 5385000 , industrielle Maschinen im Gesamtwert von DM 13065000 und andere Erzeugnisse für weitere DM $154000 .{ }^{40}$ Das AJDC seinerseits importierte vor allem Arzneimittel und anderes medizinisches Material sowie diverse Güter für den sozialen Bereich. Die deutsche Ausfuhr nach Israel von für den internationalen Markt bestimmten Erzeugnissen war der Nachsicht und dem guten Willen amerikanischer und deutscher Beamter zu verdanken. Dadurch sparte Israel Hartwährung, die nun für dringend benötigte andere Güter und Leistungen verwendet werden konnte. Bedeutend unnachgiebiger zeigten sich die Besatzungsbehörden im Falle einer - schlampig organisierten - Großbestellung für die israelische Eisenbahn, die für den Export gesperrte Güter enthielt und bei der selbst Adenauers persönliche Intervention nichts ausrichten konnte. ${ }^{41}$

Ein weiterer Einfuhrkanal aus Deutschland war der von der Regierung autorisierte reguläre Import gegen Hartwährung, zum Teil zu militärischen Zwecken. Doch öffentlicher Protest, auch in der Knesset zum Ausdruck gebracht, zwang die Behörden, diesen Import einzuschränken und den Boykott zu verschärfen wenn auch mit geringem Erfolg. Offizielle Quellen beziffern die Einfuhr aus Deutschland in den Monaten September und Oktober $1949 \mathrm{zwar}$ nur auf IL 757000 , ein äußerst geringer Betrag. Doch ob die offizielle Statistik auch all die erwähnten klandestinen Importkanäle einschließt, darf bezweifelt werden. Nicht nur Einzelpersonen, auch große Unternehmen, wie etwa die israelische Baufirma Solel-Boneh, handelten mit der Bundesrepublik Deutschland ohne offizielle Ge-

40 Goldmann im Namen von JAFP an JRSO vom 7. 10. 1953, YIVO, AJC, RG 347, GEN10, Box 292.

41 Der Transportminister an Landauer vom 28. 10. 1949; Landauer an Lipschitz vom 5. 1. 1950, CZA, S35/150, N.L. Lipschitz. 
nehmigung. ${ }^{42}$ Auch der israelische Export nach Deutschland vollzog sich abseits regulärer Pfade. So erreichten israelische Zitrusfrüchte, das einzige israelische Exportprodukt zu jener Zeit, den deutschen Markt über holländische, dänische und finnische Kanäle, ohne in der offiziellen israelischen Statistik als Export nach Deutschland ausgewiesen zu werden. ${ }^{43}$ Aus alledem geht hervor, daß der Grundstein des kommerziellen Austauschs zwischen der Bundesrepublik Deutschland und dem Staat Israel zwischen 1946 und 1953 gelegt wurde, trotz offiziellen Boykotts. Der Koreakrieg wirkte sich negativ auf die Handelsbeziehungen zwischen beiden Ländern aus, was darauf zurückzuführen ist, daß die westdeutsche Industrie Militär- und Kriegsgüter nun auf dem Weltmarkt verkaufen konnte. Der industrielle Aufschwung der Bundesrepublik und möglicherweise auch die wachsenden Außenmärkte bewirkten eine zusätzliche Verringerung des westdeutschen Interesses am israelischen Markt, das Mendelssohn vergeblich mit dem Angebot zu beleben versuchte, Entschädigungsgelder für die Einfuhr deutscher Güter zu verwenden. ${ }^{44}$

Wie aus obiger Darstellung hervorgeht, spielten beim deutschen Export nach Israel sowohl wirtschaftliche als auch politische Anreize mit. Vor dem Koreakrieg suchte die Bundesrepublik Deutschland Märkte, um die Beschäftigung anzukurbeln und brachliegende Industrien zu beleben. Ein Trend, der zumindest hinsichtlich gewisser Produkte auch nach Einsetzen des Wirtschaftsaufschwungs noch anhielt. Mendelssohn legte in Deutschland eine Güterbestelliste vor, die aufmerksam studiert wurde. Deutsche Firmen erkundigten sich bei israelischen Vertretern über Exportmöglichkeiten nach Israel und boten verschiedene Produkte an. Doch der Mendelssohn-Plan scheint nicht der einzige israelische Vorstoß zur Förderung des gegenseitigen Handels gewesen zu sein. Gemäß den Akten des Auswärtigen Amts machte der Schweizer Bankier H. Seligmann [?] der Handelspolitischen Abteilung des Auswärtigen Amts am 4. April 1951 das Angebot, einen Warenauscausch zwischen der Bundesrepublik Deutschland und Israel zu arrangieren. „Zum Unterschied vom Mendelssohn-Plan wird offenbar bei diesem Plan zunächst ein echter Warenaustausch angestrebt" ${ }^{\text {, }}$, bemerkte dazu ein Vertreter des Auswärtigen Amts. ${ }^{45}$ Nach Seligmann wurde ein gewisser G. Farber ${ }^{46}$ in der handelspolitischen Abteilung des Auswärtigen Amts vorstellig. Der als „erster inoffizieller Vertreter Israels und Wirtschaftsberater der israelischen Ministerpräsiden-

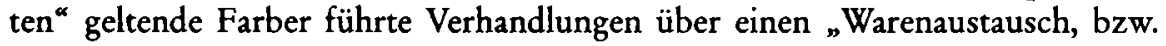

42 KNESSET-Protokolle, 1. Knesset, 1. Sitzung am 9. 1. 1950, S. 109; Interview mit Gershon Avner am 2. 9. 1986, BGA, Oral History Division, Testimony.

43 Becker an Hallstein vom 1.3. 1952, PA, 244-13/II, 306/52.

44 Ausarbeitung von Kurt Mendelssohn "Israel als Markt für Industrieerzeugnisse“ vom 20. 4. 1950, CZA, S35/70; RhEINISCHER MERKUR vom 27. 1. 1951; Mannesmann-Export, Düsseldorf, an M. Kempinski $\mathrm{GmbH}$,, betr. Export von Leichtmetallrohren nach Israel vom 9. 4. 1951, CZA, S35/142.

45 Vermerk des AA betr. Der gegenwärtige Stand der Israel-Frage in Bezug auf Deutschland vom 10. 4. 1951 (in Abschrift), PA, 210-01/35, Bd. 2.

46 Es könnte sich um das Knessetmitglied und Mitglied des Ministerrats für wirtschaftliche Angelegenheiten Herbert Yeshayahu Foerder (Progressive Partei) gehandelt haben. Der in Deutschland geborene Foerder war verschiedentlich in Deutschland unterwegs. 
über ein entsprechendes Abkommen". Sein Angebot beruhte auf den israelischen „Restitutionsforderungen“ in der Höhe von anderthalb Milliarden Dollar. Als in Frage kommende Importgüter nannte er vorfabrizierte Häuser, Arzneimittel, Zinnprodukte und Röhren, d. h. Produkte, die bereits auf der israelischen Importliste von Deutschland standen. Farbers Angebot wies indessen folgende Neuerung auf: Er offerierte den Export von Zitrusfrüchten und Pottasche und bat um einen Kredit über 5 Millionen Dollar. ${ }^{47}$ Farber regte an, den Warenaustausch über private Firmen abzuwickeln, um empfindliche Reaktionen der israelischen Öffentlichkeit zu vermeiden.

Die deutschen Vertreter erkannten rasch, daß hier eher ein politisches als ein kommerzielles Angebot vorlag und behandelten es dementsprechend. In einer internen Beratung unter Blankenhorns Teilnahme wurde bemerkt, daß Israel außer Zitrusfrüchten kaum etwas zu bieten habe, während es selbst teure Güter aus Deutschland zu importieren wünsche. Deutschland könne, so wurde weiter ausgeführt, als Geste des guten Willens israelische Orangen kaufen, doch der ganze Vorschlag sei von seiner politischen Seite zu beurteilen. Der israelische Vorstoß demonstriere den Willen, die Barrieren bei der Aufnahme der Beziehungen mit der Bundesrepublik Deutschland zu beseitigen. Insofern waren Handelsbeziehungen als Vorstufe und Wegbereiter einer bevorstehenden deutsch-israelischen Annäherung auf politischer Ebene und einer Einigung in der Retributionsfrage zu verstehen.

\section{Die Alliierten und die Schilumim}

Zwei herausragende jüdische Persönlichkeiten außerhalb Israels, Hendrik G. van Dam, der erste Vorsitzende des Zentralrats der Juden in Deutschland (ZJD), und Robert M.W. Kempner, amerikanischer Chefankläger bei den Nürnberger Prozessen, leisteten einen wesentlichen Beitrag zur Förderung des „Reparationsgedankens" sowohl in ideologischer als auch in praktischer Hinsicht. Van Dam rief wiederholt zu "Wiedergutmachung" auf, vor allem im ZJD-Organ, der Allgemeinen Wochenzeitung der Juden in Deutschland. Daraufhin beauftragte das israelische Außenministerium van Dam Mitte 1950 über den Konsul Eliahu Livneh mit der Ausarbeitung eines Gutachtens zur Reparationsfrage. Das hieraus resultierende Dokument mit dem Titel „Das Problem der Reparationen und Wiedergutmachung für Israel" beeinflußte die israelische Haltung in dieser Angelegenheit nachhaltig. ${ }^{48}$ Van Dams Neuerung bestand im Versuch, die jüdischen und israelischen Reparationsansprüche auf eine rechtliche Basis zu stellen, d.h. sie im internationalen Recht zu verankern. Ein weiteres Merkmal seines Berichts war der Aufruf zu direkten Gesprächen zwischen Israel und der Bundesrepublik Deutschland und die Warnung vor dem „ungenutzten Verstreichen der sich bietenden Gelegenheit“.

47 Vermerk der Abteilung IV, AA, betr. Unsere Wirtschaftsbeziehungen zu Israel vom 2. 7. 1951, Becker an den Herrn Staatssekretär über Herrn Blankenhorn vom 2. 7. 1951, PA, 210-01/35, Bd. 2.

48 Vollständiger Textabdruck: Der DeUTSCh-ISRaelische Dialog, Bd. 1, S. 19-25. 
Die Perspektive des im Umgang mit deutschen Ämtern vertrauten Rechtsanwalts van Dam unterschied sich wesentlich von derjenigen der fernab in Israel lebenden Juden. Insofern kam er dem Standpunkt der anderen jüdischen Politiker außerhalb Israels nahe, die für direkte Verhandlungen zur Lösung der Reparationsfrage plädierten.

Kempner, noch von seiner Rolle bei den Nürnberger Prozessen her bekannt und seither ohne öffentliche Funktion, sprach Ende 1950 anläßlich eines Besuches in Israel, wo er sich für einen Vortrag über die Nürnberger Prozesse aufhielt, als sich die Regierung intensiv mit den Beziehungen zu Deutschland befaßte, ein deutliches Wort gegenüber israelischen Regierungsvertretern, die ihn in der Reparationsfrage konsultierten: Die Deutschen seien ernsthaft an einer Verständigung interessiert, doch diese ergebe sich nicht durch Telepathie. Man müsse miteinander reden. Er ermutigte die Israelis, beherzt und vorausschauend zu handeln. ${ }^{49}$ Kempner und van Dam wurden ernst genommen, und ihre Ratschläge verhallten nicht ungehört. Das Jahr 1951 war von intensiver Aktivität geprägt. Verschiedene aufeinander folgende Initiativen zeitigten Erfolge.

Einer der ersten israelischen Schritte im neuen Jahr betraf die Änderung der Terminologie. Der Begriff „Reparationen“ wurde durch die neue hebräische Wortprägung „Schilumim“, zu deutsch „Zahlungen“ oder „Vergeltung“, bzw. durch die englischen Bezeichnungen "Collective Indemnities" oder "Collective Recompense“, zu deutsch „kollektive Entschädigung“, ersetzt. Der Begriff „Reparationen" wurde verworfen, weil damit Zahlungen von Kriegsverlierern an Sieger gemeint waren, was in diesem Fall offensichtlich unpassend war. ${ }^{50}$ Er weckte zudem unangenehme Erinnerungen an den Versailler Vertrag. Und auch in der amerikanischen politischen Tradition wurde er nicht besonders geschätzt. Zudem fürchteten die westlichen Alliierten eine sowjetische Gegenreaktion im Falle einer Ausweitung der Reparationsfrage auf Israel und die jüdische Welt und ein damit verbundenes Wiedererwachen der Reparationsproblematik. In Deutschland weckte der Begriff „Reparationen“ ebenfalls ungute Gefühle und unangenehme Erinnerungen, was die politische Führung der Bundesrepublik dazu bewog, konsequent nur noch den Ausdruck "Wiedergutmachung " zu verwenden. ${ }^{51}$

Der Nachhall des Versailler Vertrags und der Effekt der Reparationen auf die Weltwirtschaft und Weltpolitik ließen diese Art von Zahlungen in den Augen der USA unerwünscht erscheinen. Der britische Nationalökonom John Maynard Keynes begründete hiermit "die zurückhaltende US-Politik bezüglich der Deutschland aufzuerlegenden Reparationen". Die USA, fügte Keynes unmißverständlich hinzu, seien Signatarstaat des Pariser Vertrages, wonach Deutschland von der Reparationspflicht befreit sei und die USA etwaige Reparationsansprüche gegenüber Deutschland nicht unterstützten. Die US-Regierung sei entschlossen,

49 Presse- und Informationsdienst der Bundesregierung vom 8.1. 1951, PA, 210-01/35, Bd. 2.

50 The Concise Oxford Dictionary of Current English; Columbia-Viking Desk ENCYCLOPEDIA.

51 Rainer Barzel im Gespräch mit dem Autor am 8. 11. 1989; Brief an den Autor vom 5. 1. 1991. 
die Wiederholung einer Situation zu vermeiden, in der die USA die Deutschland auferlegten Reparationen letztlich bezahlen müßten.52 Die Vereinigten Staaten waren also gemäß dieser Logik gegen Reparationen, und jeder israelische Reparationsanspruch mußte daher zwangsläufig amerikanischen Widerstand hervorrufen. Israel war deshalb gezwungen zu versuchen, Washington davon zu überzeugen, daß es sich um einen Anspruch besonderer Art und nicht um Reparationen im eigentlichen Sinne handelte bzw. Washington gegenüber den humanitären und nicht den völkerrechtlichen Aspekt dieses Anspruchs zu unterstreichen. Der Begriff „Reparationen“, so die Einschätzung, war somit kontraproduktiv. Er minderte die Chancen, Zahlungen aus Deutschland zu erhalten. In Jerusalem fiel deshalb bald die Entscheidung, künftig auf den Begriff „Reparationen “ zu verzichten.

Es war jedoch nicht einfach, einen anderen Begriff zu finden. Die in Deutschland geläufige Bezeichnung „Wiedergutmachung“ für die finanzielle Entschädigung der Opfer des Nationalsozialismus war nicht unproblematisch, da ihr leicht verletzende Konnotationen anhingen und ihr verallgemeinernder Charakter für den differenzierenden Gebrauch ungeeignet war. $\mathrm{Da}$ auch in der hebräischen Sprache ein passender Ausdruck fehlte, prägte der linguistisch gebildete und für seine besondere Vorliebe für die hebräische Sprache bekannte Außenminister Moshe Sharett Ende 1950 in Anlehnung an zwei Bibelstellen den bereits erwähnten Begriff „Schilumim“ (Vergeltung, Zahlung), der hernach in den israelischen Sprachgebrauch einging. ${ }^{53}$ Sharett soll seine Wortprägung mit folgendem Argument kommentiert haben: Die Juden hätten weder die Macht zur Vergeltung noch die Absicht dazu, doch die Deutschen sollten zumindest tausendfach Entschädigung zahlen. ${ }^{54}$ "Schilumim" stand aber nicht nur für Vergeltung oder Rache. Kaum zufällig dürfte etwa die hebräische Sprachverwandtschaft von „Schilumim“ mit "Schalom" (Friede, Ausgleich) gewesen sein, eine Andeutung des möglichen Ausgleichs mit Deutschland. In den israelischen Dokumenten, die englische Korrespondenz eingeschlosen, wurde der Ausdruck „Reparationen “ fortan vollständig durch „Schilumim" ersetzt, womit ausschließlich deutsche Zahlungen an den Staat Israel gemeint waren. 55

Hochkommissar McCloy hatte zuvor dem israelischen Botschafter in Washington geraten, den Ausdruck „Reparationen“ durch „kollektive Restitution“ (Collective Restitution) zu ersetzen, da ein israelischer Reparationsanspruch keine Duchsetzungschancen habe. ${ }^{56} \mathrm{McCloys}$ direkte Intervention bewog das israelische Außenministerium stellvertretend für die jüdische Seite, fortan von Entschä-

52 Brief von Daniel F. Margolies an den Autor vom 13.12. 1990; vgl. Brief von Jacques J. Reinstein an den Autor vom 19.7.1990.

53 5. Buch Mose 32,35: „Die Rache ist mein, ich will vergelten“; Jesaia 34,8: „Denn es kommt der Tag der Rache des Herrn und das Jahr der Vergeltung, um Zion zu rächen."

54 Brief der Tel Aviver Historikerin Dinnah Porat an den Autor vom 30. 11. 1989. Der verstorbene Vater von Dr. Porat, Moshe Kitron (Kostrinsky), war ein persönlicher Freund von Sharett und soll diese Anekdote seiner Tochter während des Eichmann-Prozesses erzählt haben.

55 Hiernach steht überall dort „Schilumim “, wo „Reparationen“ oder „Wiedergutmachung“ gemeint sind.

56 Avner an Livneh vom 7. 7. 1951, ISA, 534/4a. 
digung (Recompense) statt von Reparationen (Reparations) zu sprechen. ${ }^{57}$ Die neue Sprachregelung brachte insofern auch inhaltlich eine Änderung, als sie signalisierte, daß die Juden auf Reparationen verzichteten, dafür nun aber ein Recht auf Entschädigung geltend machten.

Der Beschluß der israelischen Regierung vom 3. Januar 1951 bezüglich der offiziellen Note an die vier Großmächte wurde von den leitenden Beamten des israelischen Außendienstes mit Zurückhaltung aufgenommen. ${ }^{58}$ Nicht alle höheren Beamten des Außenministeriums unterstützten direkte Gespräche mit Deutschland. Pragmatikern standen Stimmen gegenüber, die Schmerz und Bitterkeit zum Ausdruck brachten. Die sich ergebende Pattsituation in der Regierung offenbarte die Spaltung im politischen Establishment in dieser Frage und verdeutlichte erstmals den Konflikt zwischen der alten, stark ideologisch geprägten Garde und der zweiten Generation, deren führende Vertreter sich vor allem im Militär und im öffentlichen Dienst profilierten. Die sogenannten Bitsuistim (hebr. für „Vollstrekker $^{\prime}$ ) orientierten sich eher an pragmatischen Vorgaben als an ideologischen Fernzielen. Die emotionsgeladene Debatte über die Schilumim und die Beziehungen zu Deutschland waren gleichsam der erste große öffentliche Auftritt der Bitsuistim. Doch selbst in den Reihen der „Alten Garde“ wurden schon Stimmen laut, die den Dialog mit Deutschland für unumgänglich hielten, so etwa der Generaldirektor des Finanzministeriums, David Horowitz, der zu der Überzeugung gelangt war, daß Westdeutschland dem Staat Israel aus seiner finanziellen Notlage heraushelfen könnte. Drei Jahre nach der Staatsgründung herrschte in Israel großer Mangel an Devisen, Erdöl und Nahrungsmitteln. Zehntausende von Neueinwanderern lebten in Zelten und waren ohne Arbeit. Horowitz suchte verzweifelt neue Einnahmequellen und stieß dabei auf die Schilumimidee. Der starke sozioökonomische Druck überzeugte schließlich auch die politische Führung von der Notwendigkeit ideologischer Kompromisse.

Im Jahre 1950 ebbte die große Einwanderungswelle aus Europa, vor allem von Holocaust-Überlebenden, langsam ab. Die Aufnahme dieser Neueinwanderer erwies sich als schwere wirtschaftliche Belastung. Beim nächsten Einwandererstrom, diesmal aus islamischen Ländern, waren die staatlichen Mittel deshalb besonders knapp. Während der Schilumimanspruch mit den Kosten für die Aufnahme von Opfern des Nationalsozialismus gerechtfertigt wurde, war es in Wirklichkeit also die Notlage der orientalischen Einwanderer, die die israelische Staatsführung zu ideologischen Konzessionen im Hinblick auf direkte Verhandlungen mit Deutschland zwang. Horowitz nimmt für sich in Anspruch, Ben Gurion und Sharett von der Machbarkeit der Schilumimidee überzeugt bzw. sie zum ersten $\mathrm{Mal}$ angeregt $\mathrm{zu}$ haben, was mehr als fraglich ist. ${ }^{59}$ Zweifellos waren aber sein Bericht und seine positive Haltung zur Schilumimidee aufgrund seiner zentralen

57 Shinnar an Eban vom 11. 10. 1951, ISA, 181/1.

58 Protokoll Nr. 311/24 über die Kabinettssitzung am 3. 1. 1951, ISA, 7263/10 (der Beschluß, in Sachen deutsche Reparationen an die Großmächte zu appellieren); Tekoa an den Generaldirektor vom 4. 1. 1951, ISA, 2482/12.

59 Shiva Jamim (Wochenendbeilage der isr. Tageszeitung Yedioth Achronoth) (Tel Aviv) vom 15. 9. 1972, S. 13-14; Amor an Sharett vom 3. 1. 1952, ISA 2417/4. 
Position innerhalb der israelischen Führung von größter Bedeutung. Er dürfte zudem bei der politischen Elite entscheidende Überzeugungsarbeit geleistet haben, nachdem sich ein beachtlicher Teil der leitenden Beamten des Außenministeriums für die Schilumim entschieden hatte.

Doch noch bevor die Ideen von Horowitz Gestalt annahmen, bildete sich ein weiterer deutsch-israelischer Gesprächskanal, diesmal über die Kontakte zwischen Adenauer und dem SPD-Bundestagsabgeordneten Jakob Altmaier. Altmaier, der jüdischer Herkunft war, erklärte in seinen Erinnerungen, er habe sich in den Bundestag wählen lassen, um einen Beitrag zum Wiederaufbau der deutschjüdischen Beziehungen zu leisten und um sich für deutsche Hilfe an den jungen jüdischen Staat einzusetzen. ${ }^{60}$ Altmaier war einer der Initianten der SPD-Interpellation im Bundestag vom 24. Januar 1951, in der kollektive Entschädigungszahlungen an die Juden in die deutsche öffentliche Diskussion gebracht wurde. Die Vorbereitung dieses parlamentarischen Vorstoßes fand in enger Zusammenarbeit mit der JAFP und dem israelischen Konsulat statt. Meinungsverschiedenheiten zwischen den beiden israelischen Instanzen über die Formulierung dürften dann auch zum Scheitern dieser Initiative beigetragen haben. In der Interpellation war gefordert worden, das bereits von der JRSO verwaltete, erbenlose Eigentum dem Staat Israel zu übertragen. Doch unglückliche Formulierungen und die kühle Aufnahme veranlaßte die SPD schließlich, den Vorstoß zurückzuziehen. ${ }^{61}$ Altmaier ließ sich aber nicht entmutigen und brach sogleich zum nächsten Versuch auf. Diesmal suchte er den Dialog mit Mitgliedern der Koalitionsparteien, einschließlich Adenauers CDU. Im Mittelpunkt seiner Gespräche stand die Schilumimidee und Israel. Am 14. März 1951 machte ihm Adenauer das Angebot, zwischen der Bundesrepublik Deutschland und Israel zu vermitteln. Seine Gespräche mit CDU-Vertretern hätten die Aufmerksamkeit des Kanzlers auf ihn gelenkt, vermutete Altmaier. Die SPD-Fraktion unterstützte dasUnternehmen, Altmaier handelte jedoch auf eigene Faust.

Der israelische Konsul Eliahu Livneh knüpfte freundschaftliche Kontakte zu Altmaier und ermutigte ihn, die Schilumimidee in seinen Gesprächen mit Bundestagsabgeordneten zu erörtern. Es sei ihm verwehrt, mit deutschen Persönlichkeiten zu sprechen, ohne sich selbst zu kompromittieren. Doch dies gelte nicht für den Kontakt zu Altmaier, der ein „innerjüdischer Dialog" sei.62

Über die Reaktion in Kreisen der Bundesregierung auf die israelischen Noten an die Alliierten vom 9. und 16. Januar sowie die später noch zu erwähnende Note vom 12. März ${ }^{63}$ äußerte sich der deutsch-jüdische Journalist Karl Marx wie

60 Ausarbeitung „Meine Arbeit und Mitwirkung am Israel Vertrag“ vom 5. 5. 1959, AdsD, Nachlaß Altmaier Nr. 7.

61 AlBRECHT, Ein Wegbereiter, S. 207-208.

62 Konsul Livneh, München, an das Referat Wirtschaft der Abteilung Westeuropa im israelischen Außenministerium vom 14. 3. 1951, ISA, 534/4/a.

63 Note des israelischen Außenministeriums an die britische Gesandtschaft in Tel Aviv vom 9. 1. 1951; Israelische Noten an die Vereinigten Staaten, Großbritannien und Frankreich betr. Rückerstattung und Entschädigung vom 16.1. 1951 und vom 12. 3. 1951, ISA, 2445/ 6; Note an die vier Besatzungsmächte betr. Reparationen. In: DoCUMENTS RELATING, Dokumente Nr. 3 und 5, S. 13-15, 20-24. 
folgt gegenüber israelischen Regierungsvertretern ${ }^{64}$ : Die Haltung des bundesdeutschen Kabinetts zu Israel sei positiv, nicht nur im Grundsatz, sondern auch im Hinblick auf die finanzielle Summe, die es als Verhandlungsbasis vorzuschlagen gedenke. ${ }^{65}$ Die Bundesregierung war offenbar bereit, die israelische Regierung als Vertreterin des gesamten jüdischen Volkes zu betrachten und als berechtigt, sämtliche jüdischen Ansprüche gegenüber Deutschland geltend zu machen. Im Anschluß an die israelischen Noten an die Alliierten waren die deutsch-israelischen Beziehungen im Frühjahr 1951 auf die Tagesordnung der Bundesregierung zurückgekehrt. Der damalige Bundesregierungssprecher Felix von Eckardt behauptet in seinen Erinnerungen, die Großmächte hätten nicht gewusst, wie sie auf die israelischen Noten reagieren sollten, und hätten Adenauer darüber unterrichtet. Daraufhin habe der Kanzler sich ungeachtet des rechtlichen Status der Bundesrepublik zu handeln entschlossen. ${ }^{66}$ Ein etwaiger Zusammenhang zwischen Altmaiers Initiative und den Aktivitäten des hohen WJC-Vertreters Noah Barou geht aus den vorliegenden Dokumenten nicht hervor. Demgegenüber steht fest, daß Barou den Weg für den Vorstoß von Altmaier geebnet hat, der dem Kanzler im Unterschied zu Barou - einen direkten Kanal zur israelischen Regierung bieten konnte.

Dem israelischen Entschluß, auf direkte Gespräche mit deutschen Regierungsvertretern einzugehen, gingen ausführliche Beratungen voraus. Auch die kühle alliierte Reaktion auf die israelischen Noten dürfte dabei eine Rolle gespielt haben. Der Diplomat Gershon Avner bemerkte dazu: „Die Haltung des amerikanischen Außenministers und des Leiters der Abteilung für deutsche Angelegenheiten entspricht der britischen und der französischen Haltung: In moralischer Hinsicht wird die Forderung [die israelischen Schilumimansprüche] gutgeheißen. Ihre praktische Durchführbarkeit ist jedoch angesichts der prekären wirtschaftlichen Lage Deutschlands zu bezweifeln. "67 Mit anderen Worten: Die alliierten Vorbehalte waren nicht grundsätzlicher, sondern praktischer Natur. In einer langen Abhandlung rief Avner dem Außenminister das amerikanische Interesse an einer Wiederaufnahme Deutschlands in die Gemeinschaft gleichberechtigter Staaten in Erinnerung. Die Schilumim könnten eine solche Rückkehr erleichtern, daher resultiere der amerikanische Druck auf Großbritannien und Frankreich. Die britische Öffentlichkeit, so der israelische Diplomat, hege möglicherweise gewisse Vorbehalte gegen die deutsche Wiederbewaffnung, doch die britische Regierung sei nicht daran interessiert, sich amerikanischen Vorstellungen zu verschließen. Frankreich biete wenig konkrete Hoffnung für Israel, obwohl es dem jüdischen Staat freundlich gesinnt sei. Avner versuchte seiner Position Nachdruck zu verleihen, indem er über ein lebhaftes deutsches Interesse an einem Abkommen mit dem Staat Israel berichtete und bemerkte, daß Israel es sich nicht leisten könne, diese Gelegenheit zu versäumen. Deutschland sei bald wieder bewaffnet und

64 Notiz von Leo Kohn über ein Gespräch mit Herrn Carl [sic!] Marx, Chefredakteur der 65 Ebd. Jüdischen Allgemeinen Zeitung [sic!] vom 20.3.1951, ISA, 533/7b.

66 ECKARDT, Lebenserinnerungen, S. 199.

67 Avner an Livneh vom 15. 4. 1951, ISA, 534/48. 
gleichberechtigt, und ob das deutsche Interesse an Schilumim dann noch anhalte, sei zu bezweifeln. 68

Doch die Gefahr des nachlassenden deutschen Interesses am Zustandekommen eines Schilumimabkommens war nicht die einzige Sorge der israelischen Regierung. Man befürchtete auch, ein international wieder aktives Deutschland könne als bedeutender Faktor der internationalen Politik mit den arabischen Staaten gegen Israel kooperieren. Ein anhaltender israelischer Bann gegen Deutschland mußte langfristig den Arabern in die Hände spielen, worauf die Entscheidung getroffen wurde, Horowitz zu Adenauer zu schicken. Dieses Treffen sollte unter Ausschluß der Öffentlichkeit stattfinden, nicht nur um seinen Ausgang nicht zu gefährden, sondern auch wegen der bevorstehenden Wahlen in Israel. Würde es publik, so die nicht unbegründete Sorge, könnte es die Wahlchancen der Regierungspartei Mapai massiv beeinträchtigen. Als passende Gelegenheit wurde Adenauers Reise nach Paris, wo die Schumanplanverhandlungen auf der Tagesordnung standen, gewählt. Das Pariser Treffen sollte die erste hochrangige deutsch-israelische Begegnung werden. Trotz seines gedrängten Zeitplanes, setzte Adenauer alles daran, den israelischen Vertreter zu treffen.

Das Treffen fand dann am 19. April 1951 in Adenauers Zimmer im Hotel Crillon in Paris tatsächlich statt. ${ }^{9}$ Beide Seiten vereinbarten, es im Falle des Durchsikkerns von Information zu dementieren. Die deutsche Seite war daran interessiert, die israelischen Forderungen aus erster Hand zu erfahren, während die Israelis konkrete Anhaltspunkte ernsthafter deutscher Verhandlungsbereitschaft erwarteten. Die israelische Seite legte Wert darauf, den besonderen Charakter ihrer Forderungen zu unterstreichen, die folgende Punkte enthielten: 1. ein öffentliches Bekenntnis zur Verantwortung für die NS-Verbrechen und deren öffentliche Verurteilung; 2. eine Verpflichtung zu kollektiver Entschädigung für das jüdische Volk in der Höhe von anderthalb Milliarden Dollar.

Es war keine leichte Begegnung. Horowitz war laut Shinnar besonders erregt gewesen. ${ }^{70}$ Später behauptete Horowitz, seine entschlossene Haltung habe Adenauer dazu bewogen, beide Forderungen zu akzeptieren. Es handelte sich wohlgemerkt um Forderungen, die vorher schon mehrmals an die deutsche Seite herangetragen worden waren. Wie dem auch sei, beide Seiten maßen der Pariser Begegnung größte Bedeutung bei. Der israelischen Regierung wurde die Unerläßlichkeit direkter Gespräche mit der deutschen Seite veranschaulicht, während Adenauer und sein politischer Stab Gelegenheit erhielten, die Bedürfnisse und Ansprüche der jüdischen Seite näher kennenzulernen, und darauf offensichtlich zu dem Schluß gelangten, daß es im deutschen Interesse liege, die jüdischen Ansprüche zu befriedigen.

Die Pariser Begegnung fand vor dem Hintergrund von Verhandlungen über den zukünftigen Status der Bundesrepublik Deutschland statt, in deren Verlauf

68 Avner an Sharett vom 26. 2. 1951, ISA, 534/4a.

69 JELINEK, Israel und die Anfänge, S. 133-134; ALBRECHT, Ein Wegbereiter, S. 208-209; SHINNAR, Bericht; ECKARDT, Unordentliches Leben, S. 199-200; ADENAUER, Erinnerungen, 1953-1955, S. $133 \mathrm{ff}$.

70 SHINNAR, Bericht, S. 25. 
Bundeskanzler Adenauer das Abkommen zur Gründung der Europäischen Gemeinschaft für Kohle und Stahl unterzeichnete, ein wichtiger Schritt zur deutschfranzösischen Annäherung und zur europäischen Einigung. Das neue Statut ermöglichte unter anderem die Gründung des Auswärtigen Amts, in dessen $\mathrm{Zu}$ ständigkeitsbereich die Schilumim und die Beziehungen zu Israel fielen. Auf den Weg der Bundesrepublik Deutschland zur Souveränität angesprochen, bemerkte Heinz Krekeler, der erste Gesandte der Bundesrepublik in den USA: „Eine entscheidende Voraussetzung aber dafür, daß die öffentliche Meinung in den Vereinigten Staaten die Bundesrepublik als Partner akzeptierte, war, unser Verhältnis zum Judentum auf eine neue Grundlage zu stellen." 71

Die Pariser Verhandlungen waren für beide Seiten ein günstiger Zeitpunkt. Am 6. März 1951 bestätigte die Bundesrepublik ihre Haftung für die Vorkriegs- und Kriegsschulden des Deutschen Reiches, ein Schritt von größter Bedeutung für die Absicht der jüdischen Seite, gegenüber Deutschland Ansprüche auf Entschädigung geltend zu machen.

Die beiden nicht auf Geldfragen eingehenden israelischen Noten vom Januar 1951 wurden vom israelischen Außenministerium bald als unzureichend eingestuft. Der juristische Beirat der israelischen Delegation bei der UNO verglich in einem Schreiben an den Generaldirektor des Außenministeriums die israelische Deutschlandpolitik mit der arabischen Haltung gegenüber Israel: Beide verlangten Entschädigung, waren aber nicht bereit, die zahlende Regierung anzuerkennen. ${ }^{72}$ Auf Sharetts Anregung hin kam das Thema im israelischen Kabinett am 8. Februar 1951 ein weiteres Mal zur Sprache, doch mit enttäuschendem Resultat: Das Außenministerium wurde angewiesen, anläßlich der Außenministerkonferenz der vier alliierten Großmächte eine dritte Note an die Großmächte zu richten und darin mit Nachdruck die Behandlung der israelischen Ansprüche zu fordern. Es war vergebliche Mühe. Die westlichen Großmächte waren, wie bereits dargelegt, nicht am Wiederaufrollen einer wie auch immer gearteten Reparationsfrage interessiert, und selbst wenn die Sowjetunion das israelische Anliegen unterstützt hätte, so wären die westlichen Minister geschlossen dagegen gewesen. ${ }^{73}$

Die Noten der drei westlichen Alliierten enthielten folgende gemeinsame Argumente: Die Schilumim würden letztlich dem amerikanischen Steuerzahler aufgebürdet. Darüber hinaus könnten sie den wirtschaftlichen Wiederaufbau Deutschlands gefährden, denn die momentane wirtschaftliche Lage Deutschlands lasse keine Zahlungen an Israel zu. Schließlich wurde auch noch das Argument vorgebracht, daB man sich in der gegebenen Situation darauf konzentrieren müsse, Deutschland in die Völkergemeinschaft zurückzuführen, damit dieser Staat bald seine Rolle als westliches Bollwerk gegen den Kommunismus erfüllen könne. ${ }^{74}$ Nicht weniger bedeutend, wenn auch weniger oft in die Diskussion geworfen, waren westliche Erwartungen, daß Deutschland einen Teil des westlichen

71 Krekeler an den Autor vom 25. 1. 1990; vgl. auch das Gespräch am 24. 1. 1990.

72 Robinson an Eytan vom 6. 2. 1951, ISA, 2417/1.

73 Memorandum yon W.D. Allen für Henderson vom 10. 3. 1951, PRO, FO 371/93515.

74 Bericht über die Besprechungen der jüdischen Organisationen mit Unterstaatssekretär Webb, Außenministerium, am 27. 4. 1951 und am 30. 4. 1951, ISA, 2482/13. 
Verteidigungshaushalts bestreiten, d.h. zum Unterhalt und zur Rüstung der westlichen Streitkräfte beitragen und die Kosten für die Besatzungstruppen in Deutschland ganz übernehmen müsse. Schließlich erwarteten die westlichen Gläubiger, wie bereits erwähnt, die Begleichung deutscher Kriegs- und Vorkriegsschulden, ein Anspruch, der frontal mit den jüdischen Forderungen zusammenprallte. Die Belastbarkeit der deutschen Wirtschaft wurde in den alliierten Hauptstädten sorgfältig abgewogen, und aufgrund der aus den Folgen des Versailler Vertrags gezogenen Lehren stellten sich die Alliierten gegen Zahlungen aus den Erlösen der laufenden Produktion.

Doch der dringende israelische Bedarf an Wirtschaftshilfe durchkreuzte die Pläne der Alliierten. Es war ihnen nicht verborgen geblieben, daß es sich bei den israelischen Ansprüchen nicht um eine rein grundsätzliche, abstrakte moralische Angelegenheit handelte. Das Gesuch der israelischen Regierung an die USA um Wirtschaftshilfe verdeutlichte die schwierige Lage des jungen Staates. ${ }^{75}$ Jüdische Persönlichkeiten machten die US-Regierung darauf aufmerksam, daß die Bundesrepublik den Vereinigten Staaten die Hilfe an Israel abnehmen könne. So wies etwa der Wirtschaftsberater der israelischen Botschaft in Washington, David Ginsberg, den amerikanischen Vize-Hochkommissar für Deutschland, Benjamin B. Buttenwieser, darauf hin, daß auch unter der Voraussetzung, daß der amerikanische Steuerzahler die Schilumim indirekt finanziere, er dadurch wiederum direkte Wirtschaftshilfe spare. ${ }^{76}$ Der amerikanische Außenminister erinnerte die amerikanischen Gesandtschaften daran, daß die Übernahme deutscher Vorkriegsund Kriegsschulden durch Bonn auch die finanziellen Folgen der antijüdischen Gesetzgebung und Maßnahmen des Dritten Reiches einschließlich der Juden auferlegten Geldstrafen und Sonderabgaben betreffe. ${ }^{77}$ Innenpolitische amerikanische Interessen kollidierten offensichtlich mit den Interessen der amerikanischen Außenpolitik in Deutschland.

Der letzte Vorwand, unter dem einige alliierte Kreise den Schilumimkomplex zu umgehen versuchten, war der noch ausstehende Friedensvertrag. Solange dieser nicht abgeschlossen sei, so argumentierten jene, solle eine Entscheidung aufgeschoben werden. Gewissen Vertretern des britischen Außenministeriums gefiel die Idee der Verschiebung, und auch dem State Department war sie nicht fremd.

75 J.F. Poster, die britische Botschaft in Washington D.C., an W.D. Allen, Politische Abteilung, London, vom 26. 3. 1951, PRO, 371/93515; Telegramm Nr. 445 von Acheson an die amerikanische Botschaft in Tel Aviv, vom 13.4. 1951, USNA, Tel Aviv, 321.9, Germany, RG 84, Box Nr. 5; W. Wilson an Lord Henderson vom 15. 4. 1951, PRO, FO 371/93515.

76 Dave Ginsberg an Eliezer (Kaplan?) betr. Bericht über das Gespräch mit dem stellvertretenden amerikanische Hohen Kommissar Buttenwieser vom 30. 6. 1951, ISA, 344/15; vgl. Protokoll von Horowitz über die Besprechung im Haus des Außenministers vom 22. 6. 1951, ISA, 344/15: „Der amerikanische Steuerzahler leistet ohnehin Wirtschaftshilfe in Millionenhöhe an manche Staaten. Warum sollte die USA dann dagegen sein, daß ein Teil der finanziellen Unterstützung für Deutschland an den Staat Israel gelangt, der die überlebenden NS-Opfer aufgenommen hat?"

77 Der Außenminister an die amerikanischen Gesandtschaften in Frankfurt, Paris und Tel Aviv vom 10. 5. 1951 USNA, Tel Aviv, 321.9, Germany RG 84, Box no. 2. 
Um den wirtschaftlichen Argumenten der Schilumimgegner zu begegnen, startete die israelische Regierung eine politische Kampagne unter der Führung des Generaldirektors des Finanzministeriums, David Horowitz, der in den Hauptstädten der drei alliierten Westmächte vorsprach und schriftliche Botschaften überbrachte. Zusätzlich zur intensiven Korrespondenz erarbeitete er zusammen mit seiner Assistentin Fanny Ginor eine Studie über die westdeutsche Wirtschaft, die eine rasche Erholung und ein massives Wachstum prognostizierte. ${ }^{78}$ Die israelische Haltung, so Horowitz in dieser Studie, habe jedoch eher auf moralischen als auf wirtschaftlichen oder völkerrechtlichen Argumenten zu beruhen. Den alliierten Vorbehalten sei also mit moralischen Rechtfertigungen - gestützt auf Wirtschaftsanalysen - zu begegnen. Er legte dar, der Lebensstandard der deutschen Bevölkerung liege auf vernünftigem Niveau. Er sei jedenfalls höher als der Lebensstandard in Israel. Horowitz bezeichnete es als obszön, daß das Volk, von dem das Verbrechen ausgegangen sei, nun „besser lebe als die Opfer“. Die Schilumim seien ein Akt historischer Gerechtigkeit und würden der deutschen Wirtschaft keinen Schaden zufügen. Horowitz kritisierte die alliierten Argumente, wonach Deutschland seine Mittel dringend zur Verteidigung, d.h. zum Wiederaufbau des Militärs und für Rüstungszwecke benötige, und fragte, warum dem ausgerechnet auf Kosten Israels Rechnung zu tragen sei. Das israelische Außenministerium übergab den Alliierten die Studie in Form eines Aidemémoire.

Die schwierigste Hürde für die Israelis war die Unumgänglichkeit direkter Verhandlungen mit Deutschland. Die Alliierten betrachteten die Bundesrepublik als selbständigen Staat mit unabhängiger Entscheidungskompetenz, und trotz alliiertem Verständnis für die großen emotionalen Schwierigkeiten der jüdischen Seite waren ohne deutsche Mitwirkung keine Fortschritte zu erwarten. Offensichtlich waren die westlichen Großmächte bestrebt, den Eindruck zu vermeiden, daß deutsche Zahlungen an die Juden erzwungen würden, um damit die Entstehung neuer „Dolchstoßlegenden “ zu vermeiden: Deutschland könne sich nur in freier Entscheidung zum Zahlen entschließen, hieß es auf alliierter Seite. Die israelische Regierung erwartete von Deutschland Buße in Form von sogenannten „moralischen Reparationen“, ein Anspruch, der auch in der israelischen und jüdischen Öffentlichkeit auf Zustimmung stieß. Von der Umsetzung dieses Anspruchs hing der jüdische Beitrag zur Rückkehr Deutschlands in die Völkergemeinschaft ab. Zunächst war jedoch Dialogbereitschaft von jüdischer und israelischer Seite gefordert. Auch in Israel hatte sich inzwischen die Einsicht durchgesetzt, daß sich keine dritte Partei finden würde, die Israel und der jüdischen Gemeinschaft diese

78 Memorandum über die Unterredung betr. jüdische Wiedergutmachungsforderungen gegenüber Deutschland vom 10. 4. 1951, USNA, Tel Aviv, 321.9, Germany, RG-84, Box no. 5; Vermerk über das Treffen des israelischen Ministers und Herrn Horowitz mit Lord Henderson vom 18.4. 1951, PRO, FO 371/93515; Gesprächsnotiz von E.A. Besthemd vom 18. 4. 1951, PRO, FO 371/93516; Fischer an Avner betr. Besuch des französischen Außenministeriums mit Horowitz vom 19. 4. 1951, ISA, 2417/2; Horowitz an Eytan vom 4. 4. 1951, ISA, 344/15; Ausarbeitung "Wirtschaftliche Bedingungen in Deutschland und die Zahlung von Wiedergutmachungsleistungen an Israel" vom 13. 5. 1951, ISA, 2482/13. 
schwierige Aufgabe abzunehmen gewillt gewesen wäre. Israel hatte die Wahl, entweder mit Deutschland direkte Gespräche zu führen und eine Entschädigung zu erreichen oder den Anspruch auf Schilumim endgültig aufzugeben.

In Jerusalem schien man nicht mehr zu zögern. Gershon Avner behauptete, die israelische Note vom 12. März 1951 sei mit stillschweigender deutscher Zustimmung übergeben worden. Sie habe bezweckt, die Alliierten davon zu überzeugen, daß Deutschland durchaus in der Lage sei, einer etwaigen Schilumimverpflichtung nachzukommen. ${ }^{79}$ Für diese angebliche deutsch-israelische Konspiration zur Beseitigung der wirtschaftlichen Vorbehalte der Alliierten gegen Schilumim liefern die Dokumente allerdings keine Anhaltspunkte. Die Alliierten legten die israelischen Noten dem gemeinsamen Dreierausschuß (IGS) zur genaueren Analyse vor, zum Nachteil der Israelis, da eine Prüfung der israelischen Forderungen im Kontext der deutschen Schulden den besonderen Charakter des Schilumimanspruchs in Frage stellte. Das Bestreben, die Schilumim schlicht den anderen deutschen Schulden hinzuzuaddieren, drohte später die Schilumimverhandlungen ganz zu gefährden. Die Begegnung im Hotel Crillon führte den Israelis klar vor Augen, daß die Gegenseite ernsthaft an Verhandlungen interessiert war, während sich auf deutscher Seite die Einsicht durchsetzte, daß die Auseinandersetzung mit „moralischer" und kollektiver Entschädigung unumgänglich war.

Vom Pariser Treffen zweifellos unterrichtet, bewahrten die Alliierten gegenüber den Forderungen der Israelis eine gewisse Distanz. Nach einem Treffen zwischen Ben Gurion und dem amerikanischen Außenminister Dean Acheson am 8. Mai 1951, bei dem der israelische Premier seinem Gegenüber mitgeteilt haben soll, Adenauer "wünsche Wiedergutmachung zu leisten und anerkenne grundsätzlich die Notwendigkeit einer Entschädigungsleistung ", notierte Acheson ungehalten: „Ich sagte dem Premierminister, daß mir nicht ganz klar sei, was mit Adenauers Bemerkungen gemeint sei, und betonte, daß etwaige deutsche Defizite von den USA gedeckt werden müßten. ${ }^{\text {"80 }}$ Adenauer nannte in seinen Memoiren die amerikanischen Vorbehalte als Grund für das Scheitern der Initiative Horowitz' ${ }^{81}$ Acheson war Israel gegenüber nicht besonders freundlich gesinnt und stellte sich gegen jede Entschädigungsvereinbarung, bis der amerikanische Präsident Harry S. Truman direkt zu Israels Gunsten intervenierte. McCloy dagegen zeigte Verständnis und guten Willen gegenüber der jüdischen Seite und wurde bald zur Schlüsselfigur im deutsch-israelisch-jüdischen Tête-à-tête. Die alliierten Noten wichen, wie erwähnt, einer klaren - positiven oder negativen - Antwort auf die israelischen Noten aus. ${ }^{82}$ Der Umstand, daß die israelische Regierung in ihrer Note vom 12. März 1951 sowohl materielle als auch moralische Wiedergutmachung forderte ( am wichtigsten für die Juden ist, daß Deutschland öffentlich Buße leistet für das große Unrecht an den Juden"), komplizierte die alliierte Ant-

\footnotetext{
79 Interview mit Gershon Avner am 30. 9. 1971, The Hebrew University, the Institute for Contemporary Jewry, Oral History Division.

80 Gesprächsnotiz betr. Besuch des israelischen Premierministers vom 8. 5. 1951, Truman Library, Papers of Dean Acheson, Box no. 66.

81 ADENAUER, Erinnerungen, 1945-1953, S. $133 \mathrm{ff}$.

82 Documents Relating, Dokumente Nr. 5-12, S. 28-36.
} 
wort. Doch schließlich einigten sich die Großmächte darauf zu bekräftigen, daß sie nicht in der Lage seien, die Bundesrepublik zu einer Politik zu zwingen, die sie nicht selbst anstrebe. Bei aller Sympathie, die man der Sache entgegenbringe, so die Alliierten, müsse man jede Intervention ablehnen. Die alliierte Note wurde, wie erwähnt, am 5. Juni 1951 übergeben, begleitet vom mündlichen Ratschlag, das direkte Gespräch mit der Bundesrepublik zu suchen. ${ }^{83}$

Der Geist der alliierten Noten reflektierte eine bedingte Ablehnung der israelischen Forderung. Die Geheimhaltung dieses diplomatischen Schrittes könnte darauf hinweisen, daß die Alliierten gern entgegenkommender reagiert hätten. ${ }^{84}$ Der Wunsch, Deutsche und Juden an den Verhandlungstisch zu bringen, und das Interesse an der Stützung der westdeutschen Wirtschaft schienen zunächst unvereinbar, doch schließlich setzte sich das politisch-propagandistische Kalkül und der moralische Faktor gegenüber den wirtschaftlichen Befürchtungen durch. Die deutsche Zustimmung zur Schilumimidee mag den Ausschlag gegeben haben, sowohl für die Haltung der Alliierten als auch für das israelische Ja zu direkten Verhandlungen.

Die Geheimhaltung der alliierten Noten vom 5. Juni 1951 veranschaulicht den Wandel in der alliierten Haltung. Diese Maßnahme sollte eine Gefährdung der direkten Kontakte zwischen Israel und Deutschland verhindern. Der israelische Diplomat Avigdor Dagan bemerkte dazu in einem Memorandum, die von den USA veranlaßte Geheimhaltung der besagten Note sei wertlos gewesen, da man deren Inhalt in Bonn mühelos aus anderer Quelle habe erfahren können. Für das amerikanische Handeln waren in Dagans Augen verschiedene Gründe denkbar: Eine Veröffentlichung hätte die amerikanische Außenpolitik noch mehr in Verlegenheit gebracht, nachdem sich das State Department bereits den Vorwurf des Opportunismus gefallen lassen mußte. Den Gegnern dieser Politik sollten deshalb keine weiteren Angriffsflächen geboten werden. Zudem standen in Israel Wahlen bevor, und der Inhalt der Noten hätte der linken Opposition in die Hände gespielt. Auch wollte man das Eintreffen der erwarteten sowjetischen Antwort abwarten. Dagan bemerkte zusammenfassend: „Anstatt auf Deutschland [...] werden sie [die Amerikaner] versuchen, auf uns Druck auszuüben. ${ }^{\text {" } 5}$ Er sollte Recht behalten, wie die Entwicklung in Washington und London zeigte. So bot etwa der Unterstaatssekretär für deutsche Angelegenheiten im englischen Foreign Office, Lord Henderson, dem Sekretär der britischen WJC-Vertretung, Alex Easterman, die Vermittlung eines Treffens zwischen Vertretern der israelischen Regierung und Adenauer an. Doch die Regierung in Jerusalem, die bereits über einen eigenen Gesprächskanal mit der Bundesrepublik verfügte, lehnte ab. ${ }^{86}$ Der niedere Beamte

${ }^{83}$ Bendor, Abteilung Vereinigte Staaten, an die diplomatischen Vertretungen Israels in den Vereinigten Staaten United vom 26. 3. 1951, ISA, 2482/13.

84 Protokoll von Royce vom 5. 7. 1951, PRO, FO 371/93516; Acheson an die Amerikanische Botschaft in Tel Aviv vom 3. 7. 1951, USNA, 321.9, Germany, RG-84, Box no. 2.

85 Memorandum betr. Aktionspläne hinsichtlich Forderungen gegenüber Deutschland nach der negativen amerikanischen Antwort vom 24. 7. 1951, ISA, 534/3.

86 Perlzweig an Easterman vom 21.6. 1951, Easterman an Perlzweig vom 26. 6. 1951, IJA, 220, Indemnification. West Germany, Easterman File. 
Dagan hatte keine Kenntnis vom Pariser Treffen und konnte demnach auch nicht wissen, daß die Amerikaner offene Türen einrannten.

In den frühen 50er Jahren konzentrierten sich die israelischen (und jüdischen) Anstrengungen vor allem auf zwei Bereiche: Einerseits galt es, die Alliierten und vor allem die Amerikaner zur Aufgabe ihrer Vorbehalte gegen den Geldtransfer aus Deutschland zu bewegen. Hinter vorgehaltener Hand signalisierte Israel unter bestimmten Voraussetzungen Bereitschaft zu direkten Gesprächen mit Deutschland, was den alliierten Druck gegen eine deutsch-israelische Annäherung noch zusätzlich verstärkte. Wie bereits dargelegt wurde, sprach sich Washington zwar nie grundsätzlich gegen deutsche Zahlungen an jüdischer Antragsteller aus, doch die Signale der US-Regierung in dieser Sache waren uneinheitlich. So zeigte sich das Hauptquartier der US-Besatzungsverwaltung in Frankfurt am Main entgegenkommender und nachsichtiger als das Außenministerium in Washington. Politische Stellen im Weißen Haus und im Senat waren zudem den Einflüssen unterschiedlicher Lobbies ausgesetzt, und selbst das amerikanische Außenministerium konnte sich nicht zu einer konsequenten, einheitlichen Haltung durchringen. Doch im Wettstreit zwischen den „Hütern der deutschen Wirtschaft “ und jenen, die in erster Linie für eine rasche Verbesserung der internationalen Stellung Deutschlands eintraten, behielten letztere die Oberhand.

Parallel zu den diplomatischen Aktivitäten gegenüber den Alliierten wirkte die israelische Regierung auf eine Verstärkung der deutschen Kompromißbereitschaft gegenüber der jüdischen Seite hin. Jerusalem bestand auf einer öffentlichen Verurteilung der NS-Verbrechen und einer klaren Verpflichtung zur Entschädigung, da eine solche Erklärung die Bereitschaft der jüdischen Öffentlichkeit zum direkten Dialog mit Westdeutschland verstärken würde. Trotz Rückschlägen waren auf beiden Ebenen Fortschritte zu verzeichnen.

In welchem Maße die innere Überzeugung der maßgeblichen Stellen in Bonn den Ausschlag für direkte Schilumimverhandlungen mit Israel gab und welchen Einfluß amerikanische bzw. britische Stellen auf diese Entscheidung ausübten, ist schwer zu beurteilen. Die französische Regierung übte jedenfalls Zurückhaltung, womöglich aus Furcht, deutsches Geld könnte nach Israel statt nach Frankreich fließen. Auf israelischer und jüdischer Seite setzte die Öffentlichkeitsarbeit für Schilumimverhandlungen erst mit großer Verzögerung ein. Ein gewisses Unbehagen im Umgang mit Deutschland war immer noch verbreitet, und die Israelis zögerten nicht, es zu artikulieren. Auch die Alliierten schienen gespalten zwischen politischen und wirtschaftlichen Interessen und dem Streben nach Normalisierung des deutsch-jüdischen Verhältnisses. Die im Krieg geweckten Emotionen waren immer noch nicht ganz abgeklungen. Zudem traten in den USA, Großbritannien, Frankreich und anderen europäischen Ländern widersprüchliche Standpunkte zur Frage der Reparationen an die Juden zutage. Nicht einmal scheinbar unbeteiligte Staaten wie Mexico, Peru, Uruguay oder Indien verhielten sich in dieser Frage neutral. Auf jeden jüdischen oder israelischen Schritt folgte umgehend eine freundliche oder feindliche, aber niemals indifferente, Reaktion der Regierungen dieser Länder. Zusätzlich machte sich in islamischen Staaten und besonders in der arabischen Welt das Bestreben bemerkbar, wieder an die traditionell guten Beziehungen zu Deutschland anzuknüpfen. Mit anderen Wor- 
ten: Weder die jüdische noch die deutsche Seite bewegten sich im luftleeren Raum. Jedes Wort und jeder Schritt in der Schilumimfrage löste ein internationales Echo aus. ${ }^{87}$

Mit Zustimmung des amerikanischen Außenministeriums gab die israelische Regierung eine Studie über die wirtschaftliche Lage in Deutschland zwecks $W_{1-}$ derlegung der Behauptung der deutschen Zahlungsunfähigkeit in Auftrag, die jedoch auf Drängen von Generaldirektor Horowitz, der zuvor selbst eine ähnliche Studie erarbeitet hatte, wieder abgebrochen wurde. Es gelang ihm überzeugend darzulegen, daß ein solcher Schritt nutzlos sein würde, da die Amerikaner ohnehin über breitere Informationskanäle verfügten und somit ein israelischer Bericht nicht ausreichend zur Geltung käme. ${ }^{88}$

Um der Behauptung zu begegnen, Deutschland verfüge über zu wenig Mittel für Schilumim, machte die israelische Regierung mit Verweis auf den Präzedenzfall der - aus israelischer Sicht - erfolgreichen Haavarah den Vorschlag, die Zahlungen in Form von Warenlieferungen abzuwickeln. Dagegen wurde von alliierter Seite eine Reihe von Argumenten vorgebracht: Zur Herstellung der für Israel bestimmten Ware wäre Deutschland gezwungen, einen Teil der Rohstoffe gegen Hartwährung im Ausland zu kaufen. Zudem verfüge Deutschland über so wenig Devisen, so daß es gezwungen sei, die eigenen Produkte selbständig auf dem Weltmarkt zu verkaufen. Ferner sei die einheimische Industrie und vor allem die von Israel besonders begehrte Produktion von Metallprodukten und Maschinen derzeit durch die Wiederbewaffnung völlig ausgelastet. Diesen westlichen Argumenten hielt die israelische Regierung entgegen, daß eine Milliarde Dollar nur ein Prozent des deutschen Bruttosozialprodukts ausmache und somit ein Lieferprogramm für Israel in diesem Umfang kaum nennenswerte wirtschaftliche Schwierigkeiten in Westdeutschland verursachen würde. Bei manchen der von Israel gewünschten Güter, wie etwa Zement, handle es sich zudem ohnehin nicht um eigentliche Exportgüter. Findige Vertreter schlugen vor, bestimmte Rohstoffe einzuführen und in Deutschland zu verarbeiten. Zuvor hatte die JAFP schon die Möglichkeit erwogen, jugoslawisches Bauxit in Deutschland zu Bewässerungsröhren verarbeiten zu lassen, die Israel dringend benötigte. ${ }^{89}$ Auf die zu erwartenden Vorteile der Schilumim für die deutsche Industrie wurde bereits an anderer Stelle eingegangen.

Wie bereits erwähnt, zog Horowitz die moralpolitische Argumentationslinie der von ihm als ungünstig erachteten wirtschaftlichen Debatte vor: Nicht der

87 Zur Historikerdebatte über die öffentliche Meinung zur Schilumimfrage etwa die zwei unterschiedlichen Darstellungen von: FREI, Die deutsche Wiedergutmachungspolitik, S. 215-230; WOLFFOHN, Die Wiedergutmachung und der Westen. In beiden Studien fehlt eine Berücksichtigung des breiteren öffentlichen Echos. Wenn der nicht als Anhänger des Zionismus geltende Präsident des Jewish Labor Committee, Adolf Held, sich öffentlich für die Schilumim einsetzte, kann daraus auf die Stimmungslage tausender jüdischer Arbeiter und indirekt auf das Echo von Millionen von Mitgliedern der American Federation of Labor geschlossen werden.

88 Horowitz an Sharett vom 27.7. 1951, ISA, 3068/18; Eytan an Sharett vom 29.7. 1951, ISA, $344 / 5$.

${ }^{89} \mathrm{CZA}, \mathrm{S} 35 / 42$. Die Akte von 1951 nimmt auf diesen Plan ausführlich Bezug. 
Raub jüdischen Eigentums durch die Nationalsozialisten, sondern das überzeugendere und effektivere Argument der notwendigen Aufnahme und Integration von Holocaustopfern in Israel sei zu betonen. Das Dritte Reich habe den jüdischen Exodus verursacht, und als Erbin des Reichs habe die Bundesrepublik Deutschland folglich für die kumulierten Kosten aufzukommen. ${ }^{90}$ Am 17. Juni 1951 fand im Hause des Außenministers eine strategische Planungssitzung vor dem Hintergrund der Note vom 12. März statt, auf der man sich auf eine Anzahl praktischer Beschlüsse einigte, die Sharett wie folgt zusammenfaßte: Das Außenministerium werde eine Deutschlandabteilung einrichten, eine internationale Konferenz jüdischer Organisationen zur Unterstützung der Schilumim anberaumen und eine Reihe von diplomatischen Schritten in die Wege leiten. ${ }^{91}$ Mit der Rückendeckung führender Politiker war die Leitung des israelischen Außendienstes nun in der Lage, die Umsetzung des großen Vorhabens in Angriff zu nehmen.

Gleichzeitig wuchsen jedoch in der Schilumimfrage die Spannungen zwischen dem Staat Israel und dem amerikanischen Außenministerium. Nachdem die israelische Regierung Bereitschaft zu direkten Gesprächen mit der Bonner Regierung signalisiert hatte, konzentrierten amerikanische Diplomaten ihre Anstrengungen auf die Förderung der deutschen Wirtschaft und auf die Befriedung der deutschen Öffentlichkeit. Einzelne Dokumente deuten etwa auf Versuche hin, Bonn vom Schilumimpfad abzubringen. ${ }^{92}$ McCloys Stellvertreter Buttenwieser, ein Mitglied des nicht zionistischen American Jewish Committee (AJC), stellte sich den israelischen Interessen aktiv in den Weg. ${ }^{93}$

Im Laufe der Zeit veränderte sich der Grundtenor der alliierten Berichterstattung in der Schilumimfrage schrittweise zu israelischen Gunsten. Offizielle Vertreter des jüdischen Staates wurden in den Hauptstädten der drei Westmächte stufenweise freundlicher empfangen, und ihre Gastgeber äußerten sich positiv zu Schilumim, zumindest im Grundsatz. Das Amt des amerikanischen Hochkommissars in Frankfurt am Main tendierte unverändert zu einer positiven Haltung in dieser Frage.

Parallel dazu berichtete Konsul Livneh über Anzeichen von gutem Willen in Bonn. Möglicherweise haben die Besatzungsverwaltung und die Bundesregierung einander in dieser Tendenz gegenseitig bestärkt. ${ }^{94}$

90 Livneh an Avner vom 13.6. 1951, ISA, 531/4.

91 Niederschrift über die Planungssitzung im Haus des Außenministers vom 22.6. 1951, ISA, 344/15.

92 Vermerk der Forschungsabteilung betr. deutsche Antworten auf die israelische Forderung nach Entschädigungen vom 5. 6. 1951, ISA, 2417/2; Vermerk der Abteilung IV, Becker, für Hallstein über Blankenhorn vom 2. 7. 1951, PA, 210-01/35, Bd. 2.

93 In einem Gespräch mit dem Autor in New York am 24. 5. 1988 wiederholte Buttenwieser seine früheren Standpunkte.

94 Livneh an Avner vom 13. 6. 1951, ISA, 533/7. 


\section{Die Vorbereitung der Erklärung im Bundestag}

Im Juni 1951 reiste der amerikanische Hochkommissar McCloy zu Konsultationen nach Washington. Im Vorfeld seiner Reise führte er Gespräche und korrespondierte mit in Deutschland stationierten jüdischen Vertretern, vor allem von der JRSO, die gegen die mit dem israelischen Reparationsanspruch begründete Geldausfuhrsperre nach Israel protestierte. Bis die Reparationsfrage gelöst sei, argumentierten die Alliierten, sei der Geldabfluß aus Deutschland zu vermeiden. ${ }^{95}$ Die jüdische Seite wies die Furcht vor massivem Geldabfluß als Begründung für die getroffene Maßnahme zurück und deutete auf ihren diskriminierenden Charakter hin. Darauf wurde die Geldausfuhrsperre auf höchster Entscheidungsebene behandelt und schließlich von McCloy persönlich aufgehoben. ${ }^{96}$

In Washington traf der Hochkommissar unter anderem mit dem israelischen Botschafter in den Vereinigten Staaten, Abba Eban, und dem AJC-Vorsitzenden, Jacob Blaustein, zusammen. Ein letztes nächtliches Treffen zwischen McCloy und Blaustein in Anwesenheit weiterer AJC-Vertreter stellte sich später als entscheidend für die weitere Behandlung der Schilumimfrage heraus. In jener langen Nachtsitzung im Juni 1951 in der Washingtoner Residenz von Botschafter Averell Harriman einigte man sich über eine Reihe wichtiger Fragen, wie Blaustein Jahre später in einem Schreiben an seinen Freund McCloy rekapitulierte:

${ }_{n}$ als wir uns auf eine Anzahl wichtiger Schritte einigten, die von Kanzler Adenauer und der Bundesregierung zu verlangen seien, einschließlich Restitution und Entschädigung. Sie haben daraufhin nach Ihrer Rückkehr nach Deutschland erfolgreich bei Adenauer interveniert, damit er diese Haltung annehme. Darauf machte er seine historische Erklärung, wonach sich Deutschland zur Verantwortung für die Verbrechen des Nationalsozialismus bekennen müsse und dazu verpflichtet sei, alles in seiner Macht stehende zu tun, um Wiedergutmachung zu leisten, wo dies noch möglich sei."

$\mathrm{Ob}$ nun die im folgenden zu behandelnde Erklärung Adenauers ein ausschließliches Verdienst des AJC war, wie Blaustein behauptete, ist umstritten, nicht aber der Ausgang der nächtlichen Begegnung in Washington: Am 11. Juni 1951 erfuhr Goldmann von Eban, daß „die nächste oder übernächste Woche für die Schilumimfrage entscheidend sei" ${ }^{98}$ In Briefen von Ben Gurion und Eban vom Juni 1951 werden McCloy, Harriman und Blaustein wiederholt erwähnt. ${ }^{99}$ Ein Schreiben des Direktors der Westeuropaabteilung im israelischen Außenministerium, Gershon Avner, an den Konsul Eliahu Livneh enthüllt weitere Einzelheiten: Mit Berufung auf McCloy stellte Avner fest, Adenauer habe mehrmals seine Bereitschaft zu einer finanziellen Geste gegenüber Israel erklärt, doch könne es sich nur

95 Segal an Slawson vom 11. 6. 1951, YIVO, AJC, RG 341, GEN-10, Box 291; Blaustein an Eban vom 11.6. 1951, ISA, 344/18.

96 Blaustein an Eban vom 28.6. 1951, ISA, 344/18; Blaustein an Eban vom 13.7. 1951, YIVO, AJC, RG 347, Box 291.

97 Blaustein an McCloy vom 10. 9. 1965, AJC, JSX, 65-66; Seymour J. Rubin an den Autor, ohne Datum, wahrscheinlich Anfang Juni 1989.

98 Eban an Goldmann vom 11.6. 1951, ISA, 344/18.

99 Ben Gurion an einen unbekannten Freund vom 26. 6. 1951, BGA, Correspondence File; Blaustein an Eban vom 29. 6. 1951, ISA, 344/18. 
um eine kleine Summe handeln. Der Hochkommissar habe sich dazu verpflichtet, Adenauer zu einer öffentlichen Erklärung und zu einer Geste in Form einer Zahlung zu bewegen. ${ }^{100}$ Die zugänglichen Dokumente über McCloys Konsultationen in Washington enthalten keinerlei Erwähnung der Schilumimfrage, die „ein bis zwei Wochen" im Juni 1951 waren aber offensichtlich entscheidend. Während seines Aufenthalts in der amerikanischen Hauptstadt gelang es McCloy, das Konzept einer europäischen Armee mit deutscher Beteiligung auf die Tagesordnung der amerikanischen Außenpolitik zu setzen, dessen Umsetzung aber nur mit Beteiligung Frankreichs, dem wichtigsten westlichen Gegner der deutschen Wiederbewaffnung, gelingen konnte. ${ }^{101}$ McCloy wußte selbstverständlich, daß die Aufnahme der Bundesrepublik Deutschland in die Völkergemeinschaft ohne französische Zustimmung und ohne deutsch-französische Annäherung unmöglich sein würde. 102 Doch in den Augen McCloys war Europa der „entscheidende Schauplatz des Kalten Krieges" und der deutsche Beitrag zur Sicherheit Europas somit unentbehrlich. ${ }^{103}$ In einer Rundfunkansprache im Juni 1951 befaßte sich McCloy unter anderem mit der Frage, "wie demokratisch Westdeutschland sei“. Sein Befund: Es finde ein Demokratisierungsprozeß statt. 104

Dieses Thema wurde auch im Verlaufe der erwähnten Nachtsitzung mit Blaustein im Juni 1951 ausführlich diskutiert. Am darauffolgenden Tag führte der Hochkommissar die entscheidenden Gespräche im Pentagon. ${ }^{105} \mathrm{McCloy}$ und die amerikanische Diplomatie waren, aus eigener Sicht, auch auf das Wohlwollen der jüdischen Seite angewiesen, um die Demokratisierung Deutschlands und die Einbindung der Bundesrepublik in ein europäisches Verteidigungssystem voranzutreiben. Doch dieses Wohlwollen bedingte auch einen deutschen Beitrag zur Aussöhnung mit Israel. Im Rahmen der Förderung seines Konzepts befaßte sich McCloy deshalb in der Folge besonders intensiv mit dem deutsch-jüdischen Problemkreis. Inwieweit er damit die Linie des amerikanischen Außenministeriums vertrat, läßt sich nur mutmaßen. Präsident Trumans spätere direkte Weisung zwang jedenfalls auch den amerikanischen Außendienst, sich der vorgegebenen politischen Linie anzupassen.

Zu einem Land mit langer pluralistischer Tradition gehören auch divergierende außenpolitische Konzepte. Die amerikanische Deutschlandpolitik ist ein klassisches Beispiel. So war Hochkommissar McCloy formal zwar dem State Department unterstellt, doch die amerikanische Politik in Deutschland hielt sich nicht immer an die Vorgaben des State Department. Acheson soll gesagt haben, McCloy habe sich bei seiner Ernennung zum amerikanischen Hochkommissar für Deutschland "unantastbare Autoritätsprivilegien" gesichert. Ohne seine Zustimmung sei keine wichtige Entscheidung in diesem Land getroffen worden. Butten-

\footnotetext{
100 Avner an Livneh vom 7. 7. 1951, ISA, 534/4a.

101 SCHWARTZ, From Occupation, Bd. 2, S. 260.

102 Ebd., S. 84-85.

103 Ebd., S. 563.

104 Ebd.

105 Ebd., S. 559-560.
} 
wieser nannte seinen ehemaligen Chef „die Nummer Eins in Deutschland“. 106 McCloy übernahm die Pionierrolle im Schilumimprojekt, und es gelang ihm, dessen Umsetzung voranzutreiben.

Im Sommer 1951 wurde im israelischen Außenministerium offiziell ein Deutschlandreferat im Rahmen der Westeuropaabteilung eingerichtet. Es trug die etwas schwerfällige Bezeichnung „Abteilung für Schilumim für materielle Forderungen des jüdischen Volkes gegenüber Deutschland“. Die Leitung übernahm Felix E. Shinnar, ein Ökonom deutscher Herkunft und ehemaliger Beamter des israelischen Finanzministeriums. Avigdor Dagan wurde zum Stellvertreter ernannt. Shinnar war nicht Mitglied der dominanten Regierungspartei Mapai. ${ }^{107}$ Wie der Name schon sagt, lag die Hauptaufgabe des neuen Referats in der Koordinierung sämtlicher Aktivitäten zur Umsetzung der Schilumimidee.

Fast schon Ritual waren die ebenso ausdauernden wie durchsichtigen israelischen Versuche, sich dem unvermeidlichen direkten deutsch-israelischen Kontakten mit alliierter Hilfe zu entziehen. Der israelischen Staatsführung schien es an Zivilcourage zu fehlen. Zum Durchbruch der Blockaden kam es erst aufgrund von Adenauers Angebot zu direkten Verhandlungen. Doch auch danach gingen hinter den Kulissen die israelischen Versuche weiter, den deutsch-israelischen Dialog zu bremsen, dessen Befürworter unter den israelischen Diplomaten zögerten, ihren Standpunkt den politischen Entscheidungsträgern gegenüber offen und mit Nachdruck darzulegen, und die Politiker waren froh über jede sich bietende Gelegenheit, das Unvermeidliche hinauszuschieben. ${ }^{108}$

Erst das beherzte und umsichtige Verhalten des Beamten Gershon Avner leitete die Wende ein. Am 15. August 1951 erteilte er der Ersten Sekretärin der israelischen Botschaft in Washington, Esther Herlitz, die Weisung, die Amerikaner aufzufordern, eine Erklärung der moralischen Unterstützung des israelischen Schilumimbegehrens vorzubereiten. Gleichzeitig klärte er Herlitz darüber auf, daß die Regierung in Jerusalem im Grundsatz direkte Verhandlungen mit der Bundesrepublik Deutschland unter folgenden Bedingungen bereits gebilligt habe: 1 . Die Bundesregierung müsse sich bereit erklären, die NS-Verbrechen in einer öffentlichen Erklärung zu verurteilen; 2. die Bundesregierung müsse sich verpflichten, alles in ihrer Macht Stehende zu tun, um eine Wiederholung des Holocaust von deutschem Boden aus zu verhindern; 3 . die Bundesregierung müsse sich öffentlich dazu verpflichten, wiedergutzumachen, was wiedergutgemacht werden könne, indem sie das individuelle Entschädigungsverfahren beschleunigt und sich grundsätzlich zur kollektiven Entschädigung des jüdischen Volkes bekennt; 4. der Staat Israel müsse zu direkten Verhandlungen über diese Angelegenheiten geladen werden. ${ }^{109}$ Avner verfügte zu diesem Zeitpunkt bereits über Informationen, daß

106 Interview mit Benjamin J. Buttenwieser, 121, Columbia Oral History Project.

107 Rundbrief von Eytan vom 8. 7. 1951, ISA, 2417/2; Shinnar an Livneh vom 3. 8. 1951, ISA, 181/1. Shinnar erwähnt in seinen Memoiren den 1. Juli 1951 als Datum der Gründung des Deutschlandreferats, während in den Dokumenten des Außenministeriums vom 1. August 1951 die Rede ist.

108 Vgl. kritisches Schreiben von Moshe Keren, Mitarbeiter der israelischen Botschaft in Washington, an Felix E. Shinnar vom 15. 8. 1951, ISA, 344/15.

109 Avner an Herlitz vom 15. 8. 1951, ISA, 534/4b; vgl. A.K. Helm von der britischen Bot- 
McCloys Druck auf Adenauer erfolgreich gewesen und eine deutsche Erklärung in Vorbereitung sei. Die israelische Seite verlangte Einsicht in diesen Entwurf, um Korrekturwünsche anbringen zu können.

Avners Schreiben machte eines deutlich: Die neuen israelischen Bedingungen für direkte Verhandlungen mit der Bundesrepublik Deutschland waren nahezu identisch mit den Bedingungen der bislang geheimgehaltenen Horowitz-Initiative. Wie ist also die ambivalente Haltung des israelischen Kabinetts zu erklären? Sich gegen direkte Verhandlungen aussprechen und gleichzeitig dafür stimmen? Möglicherweise hegte man in israelischen Regierungskreisen noch Hoffnung, daß die Großmächte ihre Haltung ändern und die Deutschen - ohne direkte Verhandlungen zwischen Israel und der Bundesrepublik - zum Zahlen zwingen könnten. Nachdem sich diese Aussicht endgültig verflüchtigt hatte, faßte die israelische Regierung den erwähnten Geheimbeschluß. Von den neuesten Entwicklungen nach dem geheimen Pariser Treffen nicht ins Bild gesetzte Beamte und Politiker, verfolgten indessen ihre Tätigkeiten gemäß alter politischer Leitlinien weiter. Der Diplomat Moshe Keren, verantwortlich für die deutschen Angelegenheiten in der israelischen Botschaft in Washington, war über das obige Schreiben zutiefst verärgert. Er kritisierte den unzureichenden Informationsfluß und das Fehlen einer lenkenden Hand. Doch der Rubikon war bereits überschritten und das Votum für direkte Verhandiungen in der amtsinternen Korrespondenz nun kein Geheimnis mehr. Die Öffentlichkeit wurde noch im Dunkeln gelassen, und der Deutschlandboykott blieb vorläufig eine unerschütterliche Maxime der israelischen Politik. Doch die Voraussetzungen für die öffentliche Diskussion über direkte Verhandlungen mit Deutschland waren nun geschaffen.

Die öffentliche Erklärung, die Israel von der Bundesregierung verlangte, oder die "moralische Wiedergutmachung ", wie sie in Deutschland bereits genannt wurde, begann nach dem Pariser Treffen Gestalt anzunehmen. Adenauer war ein erfahrener Staatsmann und Meister seines - politischen - Fachs: Um die Jahreswende 1950-1951, als die Alliierten und die Bundesrepublik Deutschland ernsthafte Verhandlungen über die Revision des Besatzungsstatuts aufnahmen, die zur eingeschränkten Souveränität der Bundesrepublik Deutschland führten, faßte er offenbar den Entschluß, sich auch mit dem finstersten Erbe des Dritten Reiches auseinanderzusetzen, dem Holocaust. ${ }^{110}$ Bestrebt, die hinderliche moralische $\mathrm{Hy}$ pothek auf dem Weg zurück in die Völkergemeinschaft abzutragen, wohl auch aus Schuld- und Schamgefühlen, nahm der Kanzler in Angriff, was er als „Ehrensa-

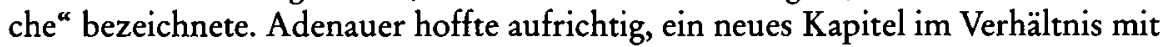
dem jüdischen Volk aufzuschlagen. Er glaubte an eine baldige Versöhnung und Verständigung. Doch Adenauer und manche Personen in seinem Umkreis unterschätzten das Trauma der jüngsten Geschichte für die jüdische Seite, was mitunter später zu Enttäuschungen führte, wenn sich gewisse Erwartungen nicht erfüllten.

schaft in London an Herbert Morrison in London, ISA, 2413/2. Helm zitiert Avner wie folgt: „Nun ist ein deutscher Schritt geboten, zum Beispiel eine Erklärung des Bundeskanzlers, daß sich die Bundesregierung zur Verantwortung für die Verbrechen gegen das jüdische Volk bekennt. "; vgl. AUERBACH, Ben Gurion and Reparations, S. $279 \mathrm{ff}$.

$110 \mathrm{Zu}$ Adenauers Rolle in der Schilumimfrage: JelineK, Political Acumen, S. 162-180. 
Die jüdische Gemeinschaft war innerlich nicht reif für die Versöhnung mit Deutschland, zumindest nicht in diesem frühen Stadium. Statt dessen erging 1951 von verschiedenen Seiten die - bereits vorher gelegentlich gestellte - klare und deutliche Forderung an die Bundesregierung, die NS-Verbrechen zu verurteilen und sich öffentlich zur Wiedergutmachung zu bekennen. Der deutsche Generalkonsul in London, Hans Schlange-Schöningen, berichtete seinen Vorgesetzten am 2. Juni 1951: „Ich darf daher die gegebene Anregung zu einer prinzipiellen Erklärung der Bundesregierung nochmals dringend in Erinnerung bringen. ${ }^{\prime 111}$ Die Londoner Times bedauerte, daß „die deutsche Regierung bis dahin kein einziges Wort der Scham oder des Bedauerns von sich gegeben hatte" ${ }^{\text {" }} 112$ Der Journalist Ernst Friedlaender sagte in einer Sendung des Nordwestdeutschen Rundfunks (NWDR) in Hamburg:

"Wäre es nicht fast selbstverständlich gewesen, offen und öffentlich auszusprechen, daß das
neue Deutschland mit Asche und Grauen an das nationalsozialistische Verbrechen denkt und
mit jener Scham, die daher kommt, daß das Geschehene mit dem deutschen Namen verbun-
den bleibt. Aber diese feierliche Erklärung der Regierung oder des Parlaments ist ausgeblie-
ben. 1113

Kurz darauf rief Erich Lüth, ebenfalls Journalist aus Hamburg, die Aktion „Frieden mit Israel“ ins Leben. ${ }^{114}$ Beide Journalisten begründeten ihre Aktionen mit dem Schweigen der Bundesregierung: Wenn die Regierung schweige, müsse das Volk seine Meinung sagen. In einem Interview mit dem Chefredakteur der New Yorker Emigrantenzeitung Aufbau, Manfred George, vom 20. Juli 1951 sprach sich Bundespräsident Theodor Heuss energisch für eine öffentliche Erklärung der Bundesregierung aus und fügte hinzu, daß der Kanzler dies auch tatsächlich erwäge. 15

Unerwarteten Beistand erhielt die Schilumimidee durch den vielbeachteten Finanzierungsvorschlag von Hjalmar Schacht, dem ehemaligen Reichsbankpräsidenten und Reichswirtschaftsminister des Dritten Reichs. Schachts Plan verband zwei Probleme und sollte die Lösung beider gestatten, nämlich den Transfer von Restitutionsgeldern und die Kapitalbeschaffung für den Wiederaufbau der demontierten deutschen Handelsflotte. Den Restitutionsgläubigern sollten verzinsliche, marktfähige und legal transferierbare Valutabonds angeboten werden, was angesichts dem anderweitig nur mit hohen Verlusten möglichen - illegalen Transfer von Restitutionsgeldern beiden Seiten Vorteile gebracht hätte.116 Schachts Vorschlag signalisierte alternative Möglichkeiten der Schilumimfinanzierung ohne amerikanische Steuergelder. Er stellte erstarrte Konzepte in Frage und trug dazu bei, die Schilumim in den Augen der breiten Öffentlichkeit zu legitimieren. Der Zeit war reif für eine deutsche Initiative.

111 Schlange-Schöningen aus London an das Auswärtige Amt in Bonn vom 2. 6. 1951, PA, 244-13 II 151.

112 The Times (London) vom 10. 7. 1951.

113 Abschrift des Wortlautes einer Radiosendung des NWDR vom 11. 7. 1951, ISA, 2482/13.

114 LÜTH, Die Friedensbitte, S. 112-115.

115 AuFBAU vom 20. 7. 1951.

116 Schacht, Hjalmar: Restitutionsgelder für den Schiffbau. In: Die ZEIT vom 8. 3. 1951. 


\section{Rosch Ha-Schana ${ }^{117} 1951$}

Der Kreis um Barou, Marx und Altmaier sowie Blankenhorns Vermittlungsbemühungen erwiesen sich als besonders hilfreich bei der Förderung der Schilumim. In seiner Antwort auf ein von Karl Marx angeregtes Schreiben des Vizekanzlers Franz Blücher schrieb Bundeskanzler Konrad Adenauer unter anderem: „Ich suche nach einer Gelegenheit, namens des Kabinetts eine Erklärung zur Judenfrage abzugeben. Man muß doch eine passende Gelegenheit haben, damit die Sache nicht so an den Haaren herbeigezogen aussieht." 118

$\mathrm{Daß}$ die Vorbereitungen für eine solche Erklärung bereits im Gange waren, ist etwa daran ersichtlich, daß Barou am 14. Juli 1951 Blankenhorn den ersten Erklärungsentwurf zurückgab, der vor dem oben zitiertem Schreiben verfaßt worden sein muß. Blankenhorn betonte zwar, Adenauer habe den Entwurf noch nicht gesehen und es handle sich "um eine rein private Initiative meinerseits“. Trotzdem darf man annehmen, daß Blankenhorn im Auftrag des Kanzlers handelte. ${ }^{119}$ Nahum Goldmann hat den Entwurf möglicherweise am 12. Juli eingesehen, ein paar Korrekturvorschläge angebracht und eine Entwurfskopie in sein Privatarchiv abgelegt. ${ }^{120}$ Buttenwieser war eigenen Angaben zufolge als "praktizierender Jude“ von Adenauer gebeten worden, ihm bei der Formulierung der Erklärung zu helfen. Beamte, Politiker und Vertreter der jüdischen Seite bzw. der israelischen Regierung erhielten Einsicht in den Entwurf. McCloy bezeichnete Bundespräsident Theodor Heuss als eine der treibenden Kräfte bei der Formulierung der Erklärung. ${ }^{21}$ Buttenwieser meinte, der Kanzler habe großen Wert darauf gelegt, daß die Erklärung in der „amerikanischen Öffentlichkeit und der öffentlichen Meinung der anderen ehemaligen Feinde Deutschlands Anklang findet" ${ }^{122}$ Die israelische Seite legte besonderen Wert auf eine Einsichtnahme in den Entwurf, denn sie wollte ihn so verfaßt sehen, daß er bei der jüdischen Öffentlichkeit einen positiven Eindruck hinterlasse. Doch Jerusalem begnügte sich nicht mit den Aktivitäten von Vermittlern wie Goldmann und Barou, sondern suchte einen direkten Kanal zu Adenauer. ${ }^{123}$ Die Israelis betrachteten den von Goldmann übermittelten Entwurf als ungenügend und nahmen gleichzeitig die Dienste Buttenwiesers, Altmaiers und des amerikanischen Außenministeriums in Anspruch. ${ }^{124}$ Auch der Beitrag des Journalisten Karl Marx wurde von Jerusalem nicht geschätzt.

117 Jüdisches Neujahr, fällt auf September/Oktober.

118 Adenauer an Blücher vom 13. 7. 1951, BArch, N 1080, Bd. 79. AdENAUER, Briefe 19511953, Dok. Nr. 63, S. 83.

119 Blankenhorn an Barou vom 18. 7. 1951, IJA, Old Archives, 220.0.

120 Entwurf einer Erklärung zur „Judenfrage ${ }^{*}$ vom 14. 7. 1951, CZA, Z 6/529.

121 McCloy an Acheson vom 2. 10. 1951, USNA, Suitland, McCloy's Papers, Box 32.

122 Interview mit Benjamin B. Buttenwieser von 1979, S. 165, Columbia Oral History Project.

123 Die Abteilung Vereinigte Staaten des israelischen Außenministeriums an die israelische Botschaft in Washington vom 13.9. 1951, ISA, 116/21; Shinnar an Livneh vom 12.9. $1951,533 / 5$.

124 Avner an Herlitz vom 10.9. 1951, ISA, 344/20; Livneh an Avner vom 25. 9. 1951, ISA, 2539/2; Avner an Livneh vom 10. und 12. 9. 1951, ISA, 2539/2; Goldmann an Sharett vom 14. 9. 1951, CZA, Z6/2345. 
Meinungsverschiedenheiten im jüdischen Lager und eine gewisse Überheblichkeit der israelischen Regierung in ihrer Eigenschaft als Vertreterin eines souveränen Staates erzeugten Spannungen, die zur Enttäuschung der israelischen Regierung über den Entwurf und die Art, wie er zustande kam, beigetragen haben mögen. Die israelische Regierung befürchtete, Adenauer könnte die Erklärung verlesen, ohne sie vorher mit Israel abzustimmen. ${ }^{125}$ Schließlich schickte Adenauer den Entwurf via Altmaier dem israelischen Botschafter in Paris, Maurice Fischer, der ihn nach Jerusalem übermittelte. Goldmann und die israelische Regierung machten gewisse Änderungsvorschläge, die Adenauer teilweise berücksichtigte. Sehr zum Unwillen der israelischen Regierung nahm er nachträglich allerdings selbst noch gewisse Korrekturen vor. ${ }^{126}$ Trotz dieser Enttäuschung war man sich auf israelischer Seite dennoch über die große Bedeutung der bevorstehenden öffentlichen Erklärung für die Zukunft des Schilumimprojekts bewußt.

Auch Adenauer war mit Schwierigkeiten im eigenen Lager konfrontiert. Der starke katholische Flügel seiner Partei war mit der Schilumimpolitik unzufrieden. ${ }^{127}$ Auf die Forderung Vizekanzler Franz Blüchers, auch jüdische Vertreter außerhalb Israels an der Formulierung der Erklärung zu beteiligen, reagierte der Kanzler mit der Beiziehung von AJC-Vertretern. ${ }^{128}$ Die Bundesregierung befürwortete den Erklärungsentwurf sodann einstimmig, nachdem daran noch geringfügige Änderungen vorgenommen worden waren. ${ }^{129} \mathrm{Die}$ SPD hingegen distanzierte sich vom endgültigen Wortlaut, der als „zu lau“ und „zu wäßrig“ empfunden wurde, und legte eine eigene Erklärung vor. ${ }^{130}$

Die israelische Regierung bereitete eine entsprechende Presseerklärung vor, nachdem sie die endgültige Formulierung der deutschen Erklärung zur Kenntnis genommen und gebilligt hatte. Außenminister Sharett, unzufrieden mit dem Entwurf, formulierte eine eigene Stellungnahme. ${ }^{131}$

Die von Adenauer herbeigeführte Mitwirkung des AJC hinterließ deutliche Spuren im Text der Erklärung. McCloy vermittelte zwischen dem Kanzler und dem AJC, und AJC-Vertreter trafen sich mehrmals mit deutschen Vertretern und mit Adenauer selbst, zum letzten Mal am Vortag der Verlesung im Bundestag. ${ }^{132}$

125 Livneh an Avner vom 20. 9. 1951, ISA, $2539 / 2$.

126 Fischer an Avner vom 20. 9. 1951, ISA, 2417/3; Fischer an Avner vom 20. 9. 1951, ISA, 2539/2; Das israelische Außenministerium an die Botschaft in New York vom 25. 9. 1951; Telegramm Eytans an die israelische Botschaft in New York vom 25. 9. 1951, ISA, 3036/ 16; Tagebucheintrag Ben Gurions vom 25. 9. 1951, BGA, BGD, Hirsch an Shinnar u.a. vom 12. 10. 1951, ISA, 344/20.

127 Livneh an Avner und Shinnar vom 3. 9. 1951, ISA, 2539/2.

128 Blücher an Adenauer vom 4.9. 1951, BArch, N 1080, Bd. 7.

129 Die KabinetTPROTOKOLLE, Bd. 4: 1951, Kabinettsitzung Nr. 175 vom 26. 9. 1951, S. 662.

130 Protokoll der Fraktionssitzung vom 25. 9. 1951. In: Die SPD-FraKTION IM DEUTSCHEN BUNDESTAG, Sitzungsprotokolle 1949-1957, S. 293. Text der Erklärung in: VerHANDlungen Des Deutschen Bundestages, 1. WP, Stenographische Berichte, Bd.9, S. $6698 \mathrm{f}$.

131 Protokoll Nr. 67/711 über die Sitzung der israelischen Regierung am 26. 9. 1951, ISA, 7264/1; Telegramm Sharetts an Eytan vom 26. 9. 1951, ISA, 3063/18.

132 Blaustein an Goldmann vom 16. 10. 1951, CZA, Z6/1621; Vermerk über die Ereignisse, 
Die Begegnung zwischen israelischen und bundesdeutschen Parlamentariern Mitte August 1951 am Rande der Konferenz der Interparlamentarischen Union in Istanbul hatte entgegen der Meinung einiger Historiker indes nur geringen Einfluß auf den Wortlaut der Erklärung. ${ }^{133}$ Die Bedeutung dieser Begegnung lag eher darin, daß es sich um die erste öffentliche Begegnung $z$ wischen Vertretern beider Seiten handelte. Die Knessetdelegation kritisierte den Umstand, daß Deutschland keine Buße für die begangenen Verbrechen geleistet und sich nicht zu kollektiver Entschädigung bekannt habe. Die deutschen Parlamentarier ihrerseits versprachen zu handeln. ${ }^{134}$ Die dramatischen Umstände der Begegnung und deren unmittelbare zeitliche Nähe zur Verlesung der Erklärung im Bundestag am 27. September 1951 verleiteten Historiker zur falschen Annahme, die Konferenz hätte 1950, also ein Jahr vorher, stattgefunden. 135

\section{Wozu eigentlich eine öffentliche Erklärung?}

In der Auseinandersetzung mit der Last der Vergangenheit wurde in der deutschen öffentlichen Diskussion mehrfach der im religiös-christlichen Bereich verwurzelte Begriff „Sühne" benutzt. Sühne bedeutet neben Reue für begangene Sünden auch Verbesserung und Reparation, oder mit anderen Worten „Wiedergutmachung". In ihrem Wirken für eine Versöhnung mit dem Judentum beriefen sich führende Persönlichkeiten des öffentlichen Lebens auf die Pflicht zur Sühne und Wiedergutmachung sowie auf den eigenen moralischen und emotionalen Notstand. Der zu erwartende politische Nutzen von Vorstößen zugunsten der jüdischen Seite war freilich auch offensichtlich. Häufig wurde auf den „freiwilligen und selbstlosen "Charakter der Erklärung im Bundestag hingewiesen, und die Alliierten widersprachen nicht. Amerikanischer und britischer Druck, ausgeübt vor allem durch Hochkommissar John McCloy, war entscheidend für die Flexibilisierung der Position deutscher Politiker, Bundeskanzler Adenauer eingeschlossen. Dieser Umstand wird in der historischen Literatur und in journalistischen Beiträgen aus politischen und moralischen Gründen oder schlicht aus Selbstverherrlichung zuweilen verdrängt. Adenauer und sein Umkreis waren empfänglich für gutgemeinte diskrete Ratschläge von Freunden. ${ }^{136}$ Oft unterschätzt wurde, wie erwähnt, die Bedeutung des Drucks der SPD auf die Bundesregierung. Die re-

die der Erklärung Kanzler Adenauers vom 28. 9. 1951 vorausgingen, o.D., YIVO, AJC, RG 341, GEN-10, Box 276.

133 Vogel, Deutschlands Weg, Bd. 1, S. 27-30; Deutschkron, Israel und die Deutschen, S. $42-43$.

134 SCHMID, Erinnerungen, S. 506.

135 Der DEUTSCH-ISRAELISCHE Dialog, Bd. 1, S. 27-29.

136 Memorandum der Abteilung IV, Becker, für Hallstein via Blankenhorn vom 2. 7. 1951, PA, 210-01/35, Bd. 2; Blaustein an McCloy vom 12. 7. 1951, USNA, Suitland, McCloy Papers, Box 30; LÜTH, Die Friedensbitte, S. 32; Entwurf einer Denkschrift des AJC zur ökonomischen Wiedergutmachung jüdischer NS-Verfolgter vom September 1965, AJC, RG-1, EXD, JSX 63; Eli Rock an Leavitt u.a. vom 19. 5. 1951, CZA, 35/84; Ferencz an Rock vom 21. 9. 1951, YIVO, AJC, RG 347, Gen-1, Box 291. 
gierende Koalition und Kanzler Adenauer persönlich waren sich des gewichtigen Engagements der Sozialdemokraten für die Erneuerung des jüdischen Lebens in Deutschland und der Wiedergutmachung bewußt, und der politische Wettstreit mit der linken Opposition förderte zwangsläufig die Bereitschaft der Koalition, auf jüdische Forderungen und Bitten einzugehen und die verlangte öffentliche Erklärung abzugeben.

Der Zeitfaktor spielte auch eine Rolle. Bei den im Mai 1951 abgehaltenen Wahlen zum Landtag von Niedersachsen wurde ein beunruhigender Zuwachs rechtsradikaler Stimmen registriert. Die Erklärung im Bundestag würde somit eine günstige Gelegenheit bieten, den durch das Wahlresultat entstandenen negativen Eindruck im Ausland, wo die politischen Entwicklungen in Deutschland sehr genau verfolgt wurden, durch eine Geste des guten Willens auszugleichen, die Bundeskanzler Adenauer besonders im Hinblick auf die Vereinigten Staaten für notwendig hielt. Sie sollte dem Kanzler Sympathien in der amerikanischen Öffentlichkeit und besonders in der jüdischen Bevölkerung eintragen. Echte demokratische Kräfte und nicht Neonazis prägten das Bild der jungen Demokratie der Bundesrepublik Deutschland, und deshalb verdiene sie das Vertrauen der Welt, lautete die zu vermittelnde Botschaft. Adenauer befürchtete zudem, daß auf der anstehenden Schuldenkonferenz jüdische Bankiers zahlreich beteiligt sein würden. Der frühere Oberbürgermeister von Köln, einem ehemaligen Zentrum von Banken in jüdischem Besitz, war vom „jüdischen Einfluß" auf die amerikanische Politik und somit von der Notwendigkeit überzeugt, Wohlwollen zu erzeugen. Bei der Untersuchung von Adenauers Äußerungen zu jüdischen Angelegenheiten und zuden für die junge Bundesrepublik notwendigen Investitionskrediten stößt man fast regelmäßig auf Wendungen, die (amerikanische) Juden mit Geld assoziieren. Solche Äußerungen, gleichgültig von welchem hohen politischen Vertreter sie stammen, sind nur schwer von den bekannten antijüdischen Vorurteilen zu trennen. Kaum zu überhören waren die Hinweise auf jüdisch-amerikanische Finanzkreise im Zusammenhang mit deutschen Darlehensgesuchen im Vorfeld der Schilumimverhandlungen. Die zeitliche Nähe zwischen der Erklärung der Bundesregierung im Bundestag und der Schuldenkonferenz war also kaum Zufall.

Auch zeremonielle Aspekte waren von Bedeutung: Marx riet Blücher, einen hohen jüdischen Feiertag als geeigneten Anlaß für die wichtige Erklärung zu wählen. Mittwoch, der 27. September 1951, der Tag, an dem die Erklärung im Bundestag verlesen wurde, lag drei Tage vor dem jüdischen Neujahr $5712 .{ }^{137}$ Die israelische Regierung war über das genaue Datum nicht informiert, ging jedoch davon aus, daß die Erklärung vor Adenauers Besuch in den Vereinigten Staaten erfolgen würde.

Die Feierstunde im Bundestag, die Abgeordneten in nachdenklicher Stimmung und die Besucherempore voll mit Gästen, einschließlich israelischer Journalisten und JAFP-Vertreter, schufen den eindrucksvollen Rahmen für die Erklärung der Bundesregierung. Bundeskanzler Konrad Adenauer verlas sie in ernstem Ton, gefolgt von der SPD-Erklärung, abgegeben vom Nestor des Bundestages und ehe-

137 Blücher an Adenauer vom 4. 9. 1951, BArch, N 1080, Bd. 79. 
maligen Reichstagspräsidenten, Paul Löbe. Danach sprachen Abgeordnete anderer Fraktionen, und den Schluß der historischen Sitzung markierten zwei Gedenkminuten.

Trotz vieler Änderungen hielt sich der endgültige Wortlaut der Erklärung im wesentlichen an Blankenhorns Entwurf. ${ }^{138}$ Am Beginn der Erklärung stand der Hinweis auf den Gleichheitsgrundsatz des Grundgesetzes, dessen Normen, so der Bundeskanzler, nur wirksam werden könnten, wenn die Gesinnung, aus der sie geboren worden seien, zum Gemeingut des ganzen Volkes würden. Die darauf folgende Mißbilligung des Antisemitismus wurde durch die Zusicherung verstärkt, Kreise, die immer noch antisemitische Hetze betreiben würden, durch unnachsichtige Strafverfolgung zu bekämpfen. In der Folge wurden die „unsagbaren Verbrechen“ des Dritten Reiches verurteilt, die zu „moralischer und materieller Wiedergutmachung verpflichten“, wobei die begrenzte Leistungsfähigkeit der deutschen Wirtschaft zu berücksichtigen sei. Die Kollektivschuldthese wurde in der Erklärung abgelehnt. ${ }^{139}$

Zwei Elemente beunruhigten die Israelis besonders: Die Verneinung der Kollektivschuld, offensichtlich ein Grundpfeiler des nationalen Ethos des neuen deutschen Staates, und die diffuse Formulierung hinsichtlich der kollektiven Entschädigung. Zudem mißfiel der israelischen Regierung die Erwähnung des jüdischen Volkes vor dem Staat Israel. Versuche, die umstrittenen Passagen in letzter Minute zu ändern, waren gescheitert. ${ }^{140}$ Die beiden Elemente reflektierten Adenauers persönliche Anschauung; er hatte sie selbst formuliert. Die Erwähnung des jüdischen Volkes an der entsprechenden Stelle war offensichtlich ein Zugeständnis an das AJC und eine Geste Adenauers an die jüdische Gemeinschaft in den Vereinigten Staaten.

Eine weitere bevorzugte Zielgruppe der bundesdeutschen Politik war das deutsch-jüdische Publikum, auch wenn sich die Beziehungen zwischen der Bundesrepublik Deutschland und den Juden deutscher Herkunft nur langsam entwikkelten. Adenauers besonderes Augenmerk für die deutsch-jüdische Gemeinschaft beunruhigte die anderen jüdischen Organisationen. Man befürchtete, die Bundesrepublik könnte versuchen, die jüdischen Organisationen gegeneinander auszuspielen. Der ehemalige Berliner Oberrabbiner Leo Baeck, Vorsitzender des Council for Protection of Rights and Interests of Jews from Germany, begrüßte die Erklärung in einem offenen Brief und wurde dafür prompt kritisiert. ${ }^{141} \mathrm{Un}$ eingeschränkt positiv reagierten zudem andere, außerhalb von Israel angesiedelte

138 Eine Kopie von Blankenhorns Entwurf liegt im IJA, Old Archives, File 220 sowie im CZA, Z6/529. Zur endgültigen Version vgl. DeuTSCHLAND UND DAS JUDENTUM, S. 3-5.

139 In der 165. Sitzung am 29.9. 1951: VERHANDLUNGEN DES DEUTSCHEN BUNDESTAGES. Stenographische Berichte. 1. Wahlperiode 1949-1953. Bonn 1953, S. $6697 \mathrm{f}$.

140 Gershon Avner an Livneh vom 24.9. 1951, ISA, 2559/2; Die israelische Botschaft in Washington, D.C. an Abteilung vom 25. 9. 1951, ISA, 2063/18; Eytan an das Generalkonsulat in New York vom 15. 9. 1951, ISA, 2063/18; Telegramm Sharetts an Eytan vom 26. 9. 1951, ISA, 2063/18; Telegramm Eytans an Livneh vom 26.9. 1951, ISA, 244; Kreutzberger an Landauer vom 28. 9. 1951, CZA, A140/552; Tagebucheintrag Ben Gurions vom 25. 9. 1951, BGA, BGD.

141 JELINEK, Leo Baeck, S. 237. 
deutsch-jüdische Organisationen, ob unabhängig und/oder im Konflikt mit der JAFP oder dem Jüdischen Weltkongreß.

Die Presse im Westen, in der Bundesrepublik und teilweise in Israel reagierte in der Regel mit Genugtuung. Ihre Kritik beschränkte sich auf Einzelheiten. ${ }^{142}$ Die jüdische Presse und auch etliche israelische Zeitungen blieben dagegen mißtrauisch und brachten Ungeduld zum Ausdruck. Hinsichtlich der deutschen Haltung forderten die Kritiker Taten statt Worte, womit sie das verbreitete Mißtrauen gegenüber Deutschland wiedergaben.

Die feierliche Bundestagssitzung beendete eine Periode vorbereitender Fühlungnahmen und stellte das Signal in Richtung Verhandlungen. Während die deutsche Seite hierzu jederzeit bereit war, gab es auf israelischer Seite - sowohl auf der politischen Ebene wie in der Öffentlichkeit - immer noch Vorbehalte. Erst die Erkenntnis, daß zusätzliche Einkommensquellen für das Überleben des jungen Staates absolut unerläßlich waren und diese nur von der Bundesrepublik Deutschland bereit gestellt werden konnten, bewog schließlich die Israelis, ihre Zustimmung zu direkten Gesprächen mit den Verantwortlichen in der Bundesrepublik Deutschland zu geben. 
